Submitted to ApJ on 30 April 2012, Accepted 1 August 2012

Preprint typeset using $\mathrm{LATEX}_{\mathrm{E}}$ style emulateapj v. 5/2/11

\title{
DISCOVERY OF A VERY LOW MASS TRIPLE WITH LATE-M AND T DWARF COMPONENTS: LP 704-48/SDSS J0006-0852AB
}

\author{
Adam J. Burgasser ${ }^{1,2}$, Christopher LuK ${ }^{1}$, Saurav Dhital ${ }^{3,4}$, Daniella Bardalez Gagliuffi ${ }^{1}$, Christine P. \\ Nicholls $^{1}$, L. Prato ${ }^{5}$, Andrew A. West ${ }^{4}$ and SÉbastien LÉpine ${ }^{6}$ \\ Submitted to ApJ on 30 April 2012, Accepted 1 August 2012
}

\begin{abstract}
We report the identification of the M9 dwarf SDSS J000649.16-085246.3 as a spectral binary and radial velocity variable with components straddling the hydrogen burning mass limit. Low-resolution near-infrared spectroscopy reveals spectral features indicative of a $\mathrm{T}$ dwarf companion, and spectral template fitting yields component types of M8.5 \pm 0.5 and $\mathrm{T} 5 \pm 1$. High-resolution near-infrared spectroscopy with Keck/NIRSPEC reveals pronounced radial velocity variations with a semi-amplitude of $8.2 \pm 0.4 \mathrm{~km} \mathrm{~s}^{-1}$. From these we determine an orbital period of $147.6 \pm 1.5$ days and eccentricity of $0.10 \pm 0.07$, making SDSS J0006-0852AB the third tightest very low mass binary known. This system is also found to have a common proper motion companion, the inactive M7 dwarf LP 704-48, at a projected separation of $820 \pm 120 \mathrm{AU}$. The lack of $\mathrm{H} \alpha$ emission in both $\mathrm{M}$ dwarf components indicates that this system is relatively old, as confirmed by evolutionary model analysis of the tight binary. LP 704-48/SDSS J0006-0852AB is the lowest-mass confirmed triple identified to date, and one of only seven candidate and confirmed triples with total masses below $0.3 \mathrm{M}_{\odot}$ currently known. We show that current star and brown dwarf formation models cannot produce triple systems like LP 704-48/SDSS J0006-0852AB, and we rule out Kozai-Lidov perturbations and tidal circularization as a viable mechanism to shrink the inner orbit. The similarities between this system and the recently uncovered low-mass eclipsing triples NLTT $41135 \mathrm{AB} / 41136$ and LHS $6343 \mathrm{ABC}$ suggest that substellar tertiaries may be common in wide $\mathrm{M}$ dwarf pairs.

Subject headings: binaries: spectroscopic — binaries: visual — stars: fundamental parameters stars: individual (SDSS J000649.16-085246.3, LP 704-48) — stars: low mass, brown dwarfs
\end{abstract}

\section{INTRODUCTION}

Our current theoretical and observational understanding of star formation holds that most stars form in multiple systems, with the frequency, mass ratio distribution and period distribution of multiples varying as a function of mass and possibly formation environment (e.g., Delgado-Donate et al. 2004; Ahmic et al. 2007; Burgasser et al. 2007b; Bate 2009, 2012; Kraus et al. 2011). Dynamics play an important role in multiple formation, as both the fragmentation of collapsing cloud cores (Boss 2001) and massive circumstellar disks (Stamatellos \& Whitworth 2009; Stamatellos et al. 2011) give rise to small $\mathrm{N}$ groups that either dissolve or stabilize into hierarchical systems (Sterzik \& Durisen 2003). Numerical calculations show that both fragmentation and dynamical evolution of stellar multiples are

\footnotetext{
${ }^{1}$ Center for Astrophysics and Space Science, University of California San Diego, La Jolla, CA, 92093, USA; aburgasser@ucsd.edu

${ }^{2}$ Visiting Astronomer at the Infrared Telescope Facility, which is operated by the University of Hawaii under Cooperative Agreement no. NNX-08AE38A with the National Aeronautics and Space Administration, Science Mission Directorate, Planetary Astronomy Program.

${ }^{3}$ Department of Physics \& Astronomy, Vanderbilt University, Nashville, TN, 37235, USA

${ }^{4}$ Department of Astronomy, Boston University, 725 Commonwealth Ave Boston, MA 02215 USA

${ }^{5}$ Lowell Observatory, Flagstaff, AZ 86001 USA

${ }^{6}$ Department of Astrophysics, Division of Physical Sciences, American Museum of Natural History, Central Park West at 79th Street, New York, NY 10024, USA
}

sensitive to the initial cloud properties - geometry, density, turbulence spectrum, and radiative and external feedback - so multiplicity statistics provide important tests for star formation theory, on par with the initial mass function, stellar mass segregation and velocity distributions.

The mass dependence of multiplicity among stars is well established, with observed frequencies ranging from $>80 \%$ for OBA dwarfs (Shatsky \& Tokovinin 2002; Kouwenhoven et al. 2005; Kobulnicky \& Fryer 2007) to $\sim 30 \%$ for systems with M dwarf primaries (Fischer \& Marcy 1992; Reid \& Gizis 1997; Delfosse et al. 2004). The frequency of multiples appears to drop even further in the very low mass (VLM; $\left.\mathrm{M} \lesssim 0.1 \mathrm{M}_{\odot}\right)$ stellar and substellar regimes. Resolved imaging studies of field and cluster VLM dwarfs, based on ground-based adaptive optics (AO) and Hubble Space Telescope (HST) observations, find relatively consistent (volume-limited) binary frequencies of 10 $20 \%$ (e.g., Bouv et al. 2003; Burgasser et al. 2003, 2006c; Close et al. 2003). However, estimates for the frequency of tightly-bound spectroscopic VLM binaries (roughly $10 \%$ of currently known systems) span a broad range of 1-25\% (Maxted \& Jeffries 2005; Basri \& Reiners 2006; Kurosawa et al. 2006; Joergens 2008; Blake et al. 2010; Clark et al. 2012). As such, there remains significant uncertainty in the overall VLM binary frequency, which has a direct impact on our understanding of how brown dwarfs form in the first place (e.g., Delgado-Donate et al. 2003). There is also evidence that the orbital characteris- 
tics of multiples are mass-dependent, with VLM binaries being on average tighter $(\langle a\rangle \approx 7 \mathrm{AU}$ versus $\approx 30 \mathrm{AU})$ and more frequently composed of equal-mass components $\left(\langle q\rangle \equiv \mathrm{M}_{2} / \mathrm{M}_{1} \approx 1\right.$ versus $\left.\approx 0.3\right)$ than their solar-mass counterparts (Allen 2007). Again, these trends may be skewed by biases inherent to the known sample of VLM binaries, identified largely as imaged pairs. A complete understanding of VLM multiplicity statistics therefore requires more robust constraints on the frequency and characteristics of short-period and low- $q$ multiples (Burgasser et al. 2007b).

An alternative method for identifying VLM multiples that avoids separation biases (both intrinsic and projected) is through spectral binaries, systems with components of different spectral types whose combined-light spectra exhibit distinct peculiarities. While this technique is more commonly associated with white dwarfM dwarf pairs (e.g., Silvestri et al. 2006), spectral binaries containing late $\mathrm{M}$ or $\mathrm{L}$ dwarf primaries and $\mathrm{T}$ dwarf secondaries have also been recognized due to their unique and highly structured near-infrared spectra (Cruz et al. 2004; Burgasser 2007b; Burgasser et al. 2008a; Stumpf et al. 2008; Burgasser et al. 2010, 2011b; Gelino \& Burgasser 2010; Geißler et al. 2011). Because blended light systems can be identified at any separation, $\mathrm{M} / \mathrm{L}+\mathrm{T}$ spectral binaries can probe the very closest separations that are inaccessible to direct imaging studies ( $a \lesssim 50$ mas). An illustrative case is the M8.5 + T5 binary 2MASS J03202839-0446358 (hereafter 2MASS J0320-0446), a system independently identified as both a spectral binary (Burgasser et al. 2008a) and a radial velocity (RV) variable with a period of $0.68 \mathrm{yr}$ and primary semi-major axis $a_{1} \sin i=0.157 \pm 0.003 \mathrm{AU}$ (Blake et al. 2008, 2010). With a projected separation of $\approx 17$ mas, this system is unresolvable with current imaging and interferometric technology. Moreover, the significant difference in component magnitudes $(\Delta K=$ $4.3 \pm 0.6)$ makes this system a potential low $q$ pair. The independent constraints provided by the component classifications and RV orbit yield robust limits on the age $(\gtrsim 2 \mathrm{Gyr})$ and orbital inclination $\left(\gtrsim 53^{\circ}\right)$ of this system (Burgasser \& Blake 2009).

In an effort to confirm and characterize spectral binaries identified in low-resolution nearinfrared spectroscopy, we have identified a new VLM system exhibiting significant RV variability. The source, SDSS J000649.16-085246.3 (hereafter SDSS J0006-0852; West et al. 2008) is an opticallyclassified M9 which appears to be both a short-period $(0.4 \mathrm{yr})$ binary with M8.5 and $\mathrm{T} 5 \pm 1$ components, and a co-moving wide companion to the inactive M7 dwarf LP 704-48. Together, this system comprises the lowest-mass triple confirmed to date. In Section 2 we describe our optical and near-infrared imaging and spectroscopic observations of this system using the $1 \mathrm{~m}$ Lick Observatory Nickel Direct Imaging Camera, the 3m NASA Infrared Telescope Facility (IRTF) SpeX spectrograph (Rayner et al. 2003), the 4m Kitt Peak National Observatory (KPNO) RC spectrograph, and the 10m Keck II NIRSPEC spectrograph (McLean et al. 1998). In Section 3 we examine the properties of the SDSS J0006-0852 and LP 704-48 pair, assessing their common proper motion, distance and probability of chance alignment; as well as the age and metallicity

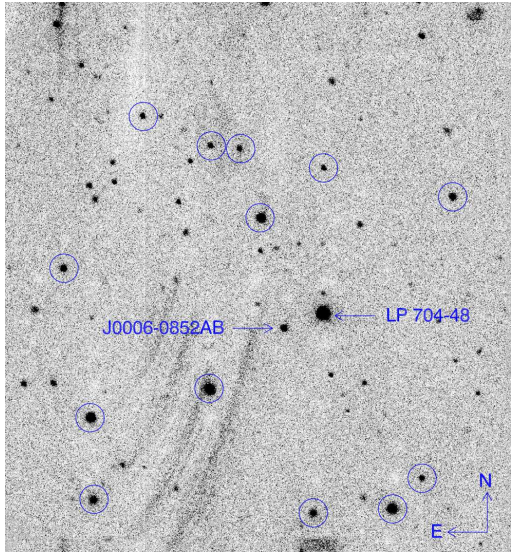

FIG. 1.- Lick/Nickel image of the LP 704-48/SDSS J0006-0852 field showing the location of the two sources (arrows and labels) and field sources used for calibrating the astrometric reference frame (circles). The field shown is approximately $5^{\prime} \times 5^{\prime}$ and oriented North up and East to the left. Streaking along the bottom of the image is due to reflected lunar light.

of both components as derived from spectroscopic and kinematic indicators. In Section 4 we analyze the lowresolution near-infrared spectrum of SDSS J0006-0852 to infer the presence of, and characterize, its $\mathrm{T}$ dwarf companion. In Section 5 we analyze our RV measurements which allow us to extract both the orbital properties (including constraints on the orbital inclination) and verify the relatively old age for this system. In Section 6 we discuss LP 704-48/SDSS J0006-0852AB in the context of other hierarchical low-mass triples, and examine whether current star formation theories or three-body interactions can create such systems. Our results are summarized in Section 7.

\section{OBSERVATIONS}

\subsection{Optical Imaging}

The SDSS J0006-0852 field was observed with the Nickel 1m CCD camera on 2011 October 28 (UT) in clear conditions with 1 .' 5 seeing. The chip was binned $2 \times 2$ for a pixel scale of $0^{\prime \prime} 37$ pixel $^{-1}$. Six dithered exposures of $150 \mathrm{~s}$ each were obtained using the $I$-band filter over an airmass range of 1.45-1.49. Dome flat and dark exposures were obtained at the end of the night for pixel calibration. Data were reduced using custom IDI7] scripts that removed the bias voltage, median-combined and normalized the flat field frames; divided the science data by the flat; masked bad pixels; generated a median sky frame that was subtracted from the science data; and registered and median-stacked the science frames to a single final image, shown in Figure 1.

Pixel positions for sources in the final stacked frame were measured using a centroiding algorithm routine attached to the atv program (Barth 2001). Using astrometry of 13 field stars drawn from the Two Micron All Sky Survey (2MASS; Skrutskie et al. 2006), we determined a linear astrometric transformation frame with residuals of $00^{\prime \prime} 3$ in Right Ascension and 0.2 and declination; i.e., $\sim 20 \%$ of the seeing radius. This transformation was used to compute coordinates for LP 704-48 and SDSS J0006-0852, listed in Table 1. Figure 2 displays the 2MASS to Nickel offsets for all of the sources in the

\footnotetext{
7 Interactive Data Language.
} 


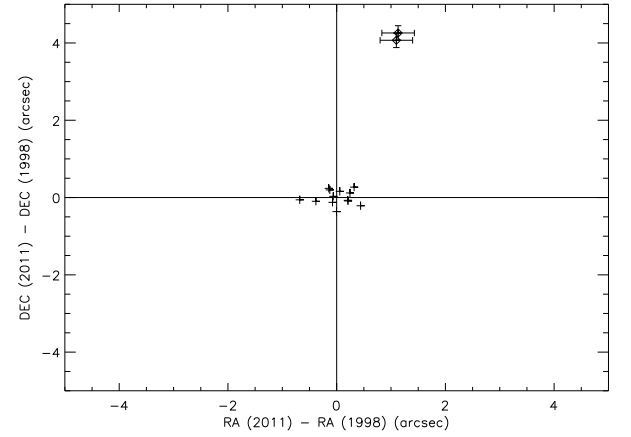

Fig. 2.- Offsets in Right Ascension and declination between 2MASS and Nickel imaging epochs for field stars (plus symbols) and LP 704-48/SDSS J0006-0852 (diamonds). The error bars on the last two data points reflect the scatter in offsets among the field stars.

TABLE 1

ASTROMETRY FOR SDSS J0006-0852 AND LP 704-48

\begin{tabular}{lcccc}
\hline \hline Source & $\begin{array}{c}\text { Epoch } \\
(\mathrm{UT})\end{array}$ & $\begin{array}{c}\alpha \\
(\mathrm{J} 2000)\end{array}$ & $\begin{array}{c}\delta \\
(\mathrm{J} 2000)\end{array}$ & $\begin{array}{c}\text { Uncertainties } \\
\left({ }^{\prime \prime}\right)\end{array}$ \\
\hline \hline \multicolumn{5}{c}{ LP 704-48 } \\
\hline POSS I $R$ & 1954.669 & 000647.66 & -085221.17 & $0.1,0.1^{\mathrm{a}}$ \\
2MASS & 1998.767 & 000647.46 & -085235.00 & $0.11,0.09$ \\
ESO $R$ & 1999.604 & 000647.45 & -085235.31 & $0.1,0.1^{\mathrm{a}}$ \\
UKST $I_{N}$ & 2000.723 & 000647.45 & -085235.66 & $0.1,0.1^{\mathrm{a}}$ \\
SDSS & 2000.740 & 000647.45 & -085235.60 & $0.06,0.04$ \\
Nickel & 2011.820 & 000647.39 & -085239.16 & $0.3,0.2$ \\
\hline \multicolumn{5}{c}{ SDSS J0006-0852 } \\
\hline 2MASS & 1998.767 & 000649.16 & -085245.70 & $0.11,0.09$ \\
ESO $R$ & 1999.604 & 000649.15 & -085245.53 & $0.1,0.1^{\mathrm{a}}$ \\
UKST $I_{N}$ & 2000.723 & 000649.14 & -085246.42 & $0.1,0.1^{\mathrm{a}}$ \\
SDSS & 2000.740 & 000649.16 & -085246.30 & $0.07,0.06$ \\
Nickel & 2011.820 & 000649.09 & -085249.96 & $0.3,0.2$ \\
& & &
\end{tabular}

REFERENCES. - POSS $1 R$, ESO $R$ and UKST $I_{N}$ astrometry are from the SuperCosmos Sky Survey (Hambly et al. 2001c|b a) ; 2MASS astrometry are from the All-Sky Data Release Point Source Cata$\log$ (Skrutskie et al. 2006); SDSS astrometry are from Data Release 7 (Abazajian et al.|2009)

${ }^{\text {a }}$ Estimated.

field. The large and similar proper motions for LP 70448 and SDSS J0006-0852 are evident, distinct from the field by $>20 \sigma$ in declination.

\subsection{Low Resolution Optical Spectroscopy}

The optical spectrum of SDSS J0006-0852 from the Sloan Digital Sky Survey (SDSS; York et al. 2000) is shown in Figure 3 compared to an M9 SDSS spectral template from Bochanski et al. (2007b). The similarity of these spectra confirms the M9 classification from West et al. (2008), although we note a slightly flatter $7500 \AA \mathrm{VO}$ band and weaker Na I lines at $8200 \AA$ for SDSS J0006-0852. Neither Li I absorption $(\mathrm{EW}<0.3 \AA)$ nor $\mathrm{H} \alpha$ emission $\left(f_{H \alpha}<4 \times\right.$ $\left.10^{-18} \mathrm{erg} \mathrm{s}^{-1} \mathrm{~cm}^{-2}\right)$ are detected in these data. Upper limits on $\log _{10} L_{H \alpha} / L_{b o l}$ were calculated by finding the $\mathrm{H} \alpha$ equivalent width corresponding to the $1 \sigma$ flux uncertainty, using the $\chi$ factor (Walkowicz et al. 2004; West \& Hawley 2008) to convert from EW to $\mathrm{L}_{\mathrm{H} \alpha} / \mathrm{L}_{\text {bol }}$ and to propagate uncertainties. We find $\log _{10} L_{H \alpha} / L_{b o l}$ $<-5.7$ for SDSS J0006-0852.

The nearby proper motion star LP 704-48 (aka
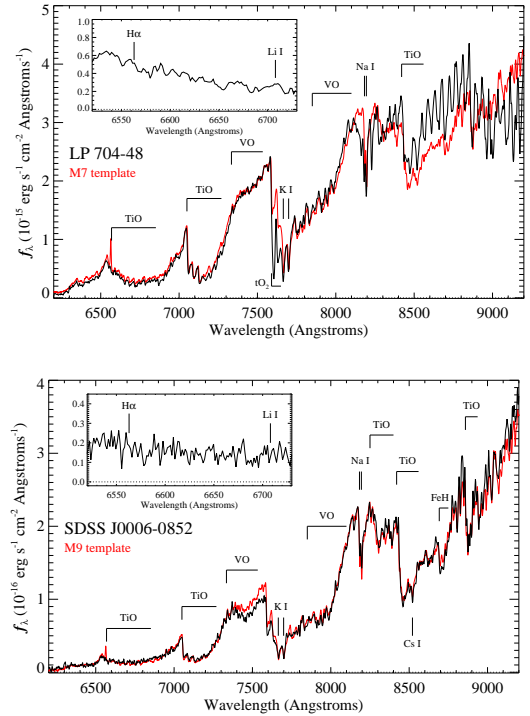

FIG. 3.- Red optical spectra of LP 704-48 (top) and SDSS J0006-0852 (bottom, from SDSS) compared to M7 and M9 SDSS spectral templates from Bochanski et al. (2007b). All spectra shown are smoothed to a common resolution of $\lambda / \Delta \lambda=1200$. Data for the two dwarfs are scaled to their apparent SDSS $i$ magnitudes, while the templates are scaled to overlap in the 7400-7500 (LP 704-48) and 8550-8600 ^ (SDSS J0006-0852) regions. Primary atomic and molecular absorption features are labeled. The inset boxes shows close-ups of the $6520-6730 \AA$ A region, showing the absence of both $\mathrm{H} \alpha$ emission and Li I absorption in both sources.

2MASS J00064746-0852350; Luyten 1980) was classified M6 by Cruz \& Reid (2002) based on low-resolution optical spectroscopy. We obtained a new optical spectrum of LP 704-48 on 2011 Nov 28 (UT) using the RC spectrograph on the KPNO $4 \mathrm{~m}$ telescope. Conditions were good, with seeing $\sim 1^{\prime \prime}$ and excellent transparency. We used a $1^{\prime \prime} .5$ slit aligned to the parallactic angle, a 600 lines $\mathrm{mm}^{-1}$ grating blazed at $7500 \AA$ (BL 420), and an order-blocking filter (OG 530) to obtain 5800-9100 $\AA$ spectra at a resolution of $\sim 2000$ and dispersion of $1.74 \AA$ pixel $^{-1}$. A single $1200 \mathrm{~s}$ exposure was obtained at an airmass of 1.32 . We observed the sdO flux standard HZ 4 for flux calibration, and obtained a set of quartz flats, bias frames, and arclamp exposures for pixel response and wavelength calibration. Data were reduced using standard routines in IRAF 8 .

The reduced spectrum for LP 704-48 is shown in Figure 3, compared to an M7 SDSS template from Bochanski et al. (2007b). Both our data and those of Cruz \& Reid (2002), most closely match this comparison source, with the exception of a downturn in flux beyond $8100 \AA$ caused by poor flux calibration in the red and strong fringing. An M7 classification is also generally consistent with spectral index-based classifications using the VO1, VO2, TiO7 and Color-M indices defined in Lépine et al. (2003, Table 2). Like SDSS J0006-0852, LP 704-48 exhibits no evidence of Li I absorption (EW $<0.13)$ or $\mathrm{H} \alpha$ emission $\left(f_{H \alpha}<3 \times 10^{-17} \mathrm{erg} \mathrm{s}^{-1} \mathrm{~cm}^{-2}\right.$; $\left.\log _{10} L_{H \alpha} / L_{b o l}<-6.2\right)$ in either our spectrum or that of Cruz \& Reid (2002). As discussed in Section 3, the lack of nonthermal emission is consistent with a relatively old age for both sources.

\footnotetext{
${ }^{8}$ Image Reduction and Analysis Facility (Tody 1986).
} 
TABLE 2

Optical SPeCtral Indices FOR LP 704-48 AND SDSS J0006-0852

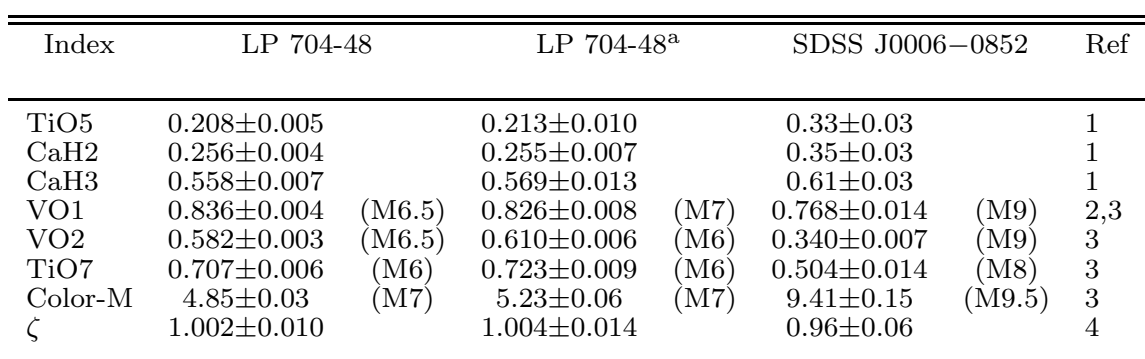

REFERENCES. - (1) Reid et al. (1995); (2) Hawlev et al. (2002); (3) Lépine et al. (2003);

(4) Lépine et al. (2007).

a Data from Cruz \& Reid (2002).

b Classifications based on the relations quantified in Lépine et al. (2003).

\subsection{Low Resolution Near-Infrared Spectroscopy}

Low resolution near-infrared spectra of SDSS J0006-0852 were obtained on 2009 November 4 (UT) with IRTF/SpeX in conditions of light cirrus and 0.6 seeing. We used the prism-dispersed mode with the 0.5 slit to obtain continuous, low-resolution $(\lambda / \Delta \lambda \approx 120)$ spectra covering the $0.7-2.4 \mu \mathrm{m}$ band. Six exposures of $120 \mathrm{~s}$ each were acquired in an ABBA dither pattern along the slit which was aligned with the parallactic angle. The A0 V star HD $1154(V=$ 8.85) was observed immediately afterward at a similar airmass for flux calibration and telluric absorption correction, and NeAr arc lamp and flat field exposures were obtained along with the standard. Data were reduced with the SpeXtool package, version 3.4 (Cushing et al. 2004; Vacca et al. 2003), using standard settings. A detailed description of our reduction procedures is given in Burgasser (2007b).

Figure 4 displays the reduced spectrum of SDSS J0006-0852 compared to equivalent data for 2MASS J0320-0446 (SpeX data from Burgasser et al. 2008a) and the M8.5 dwarf HB 2115-4518 (Hawkins \& Bessell 1988; Lodieu et al. 2005; SpeX data from Burgasser et al. in prep.). The three spectra are generally equivalent in shape, exhibiting strong TiO, $\mathrm{H}_{2} \mathrm{O}$ and $\mathrm{CO}$ bands; weaker absorption from $\mathrm{VO}$ and $\mathrm{FeH}$; and line absorption from K I and Na I (doublet lines are unresolved in these data). The spectra of both SDSS J0006-0852 and 2MASS J0320-0446 also exhibit subtle features not present in that of HB 2115-4518, most notably an excess of flux at $1.27 \mu \mathrm{m}, 1.55 \mu \mathrm{m}$ and (less prominently) $2.1 \mu \mathrm{m}$; and a distinct "notch" feature at $1.6 \mu \mathrm{m}$. As discussed in Burgasser (2007b) and shown in Section 4, these features indicate the presence of an unresolved T dwarf companion to SDSS J0006-0852.

\subsection{High Resolution Near-Infrared Spectroscopy}

High resolution near-infrared spectra of SDSS J0006-0852 and LP 704-48 were obtained on seven epochs between 2010 August and 2012 January using the NIRSPEC echelle spectrograph on the Keck II telescope. Observations are summarized in Table 3 On four epochs we used the N3 order-sorting filter and 0 . $432 \times 12^{\prime \prime}$ slit to obtain $1.18-1.30 \mu \mathrm{m}$ spectra over orders $56-66$ with resolution $\lambda / \Delta \lambda=20,000(\Delta v=$ $15 \mathrm{~km} \mathrm{~s}^{-1}$ ) and dispersion of $0.181 \AA$ pixel $^{-1}$. On 2011

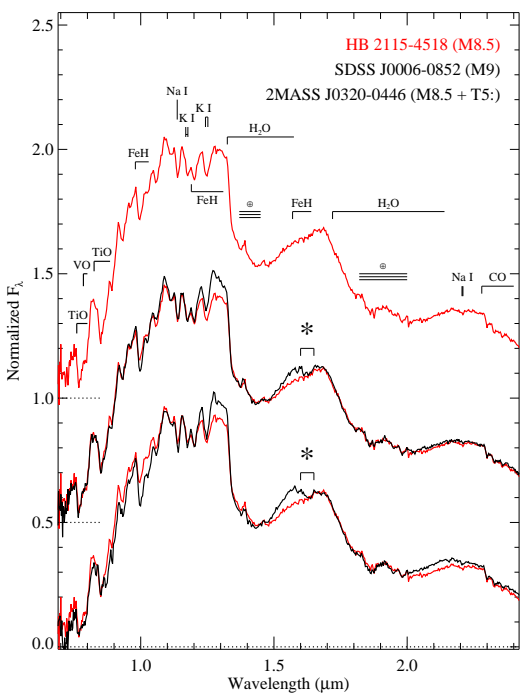

FIG. 4. - SpeX prism spectrum of SDSS J0006-0852 (center) compared to those of the M8.5 HB 2115-4518 (top; Hawkins \& Bessell 1988; Burgasser et al., in prep.) and the M8.5+T5 spectral binary 2MASS J0320-0446 (bottom; Wilson et al. 2003; Burgasser et al. 2008a). All spectra are normalized in the $1.2-1.3 \mu \mathrm{m}$ range and offset by constants. The HB 2115-4518 spectrum (in red) is also overlaid on the other two spectra for comparison. Major molecular and atomic absorption features are labeled, as is the "notch" feature at $1.6 \mu \mathrm{m}$ indicating the presence of an unresolved $\mathrm{T}$ dwarf companion. Regions of strong telluric absorption are indicated by the horizontal lines.

September 7 and 2012 January 6 (UT) we observed SDSS J0006-0852 and LP 704-48 with the N7 filter and 0 . $432 \times 12^{\prime \prime}$ slit to obtain $2.00-2.39 \mu \mathrm{m}$ spectra over orders $32-38$ with $\lambda / \Delta \lambda=20,000\left(\Delta v=15 \mathrm{~km} \mathrm{~s}^{-1}\right)$ and dispersion of $0.315 \AA$ pixel $^{-1}$. On 2012 January 15 (UT) we observed SDSS J0006-0852 with the N5 filter and $0.288 \times 24^{\prime \prime}$ slit to obtain $1.43-1.70 \mu \mathrm{m}$ spectra over orders $45-53$ with $\lambda / \Delta \lambda=30,000\left(\Delta v=10 \mathrm{~km} \mathrm{~s}^{-1}\right)$. Echelle and cross-dispersion gratings were set to the values listed in Table 3. which varied slightly from run to run to maintain consistent projection of arclamp images on the detector. We also observed seven $\mathrm{L}$ dwarfs with previously measured radial velocities from Blake et al. (2010) to serve as radial velocity standards (Table ??). For each source, we obtained spectra in AB or ABBA nodding sequences. We also observed a nearby A0 V star for flux calibration and telluric correction, 
with the exception of some N5 observations for which a comparison star was not required (see below). Dark, quartz lamp and NeArXeKr arc lamp frames were obtained at the beginning or end of each night without changing the instrument configuration.

All data were reduced using REDSPEC, an IDL-based software package developed at UCLA for NIRSPEC by S. Kim, L. Prato, and I. McLear9. Images were first corrected for pixel-response variations using the darksubtracted flat field frames. Individual orders were then isolated, rectified, and pair-wise subtracted, and spectra were extracted by summing across rows. In this study, we focused exclusively on order $59(1.283-1.300 \mu \mathrm{m})$ in the N3 data, order $49(1.545-1.567 \mu \mathrm{m})$ in the N5 data, and order $33(2.291-2.326 \mu \mathrm{m})$ in the N7 data. The latter two orders are relatively devoid of telluric absorption features (Prato et al. 2002; McLean et al. 2007), while order 33 samples the strong CO band around $2.3 \mu \mathrm{m}$ (Blake et al. 2008). Image rectification and wavelength calibration were performed using telluric $\mathrm{OH}$ emission lines present in the long science exposures for orders 49 and 59 (Rousselot et al. 2000); for order 33, we used the arc lamp images and a vacuum line list from the National Institute of Standards and Technology (NIST) atomic spectral line database (Ralchenko et al. 2011). For each observation, wavelength solutions were corrected for barycentric motion. Flux calibration and telluric absorption corrections (except for order 49) were calculated from the A0 V spectra assuming a $9480 \mathrm{~K}$ blackbody. No filtering of fringing was performed.

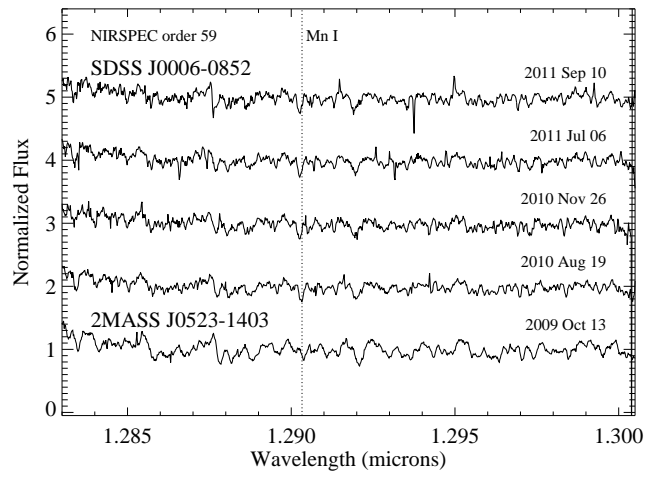

FIG. 5. - Four epochs of NIRSPEC order 59 (N3) spectra of SDSS J0006-0852 (top) compared to the L2.5 radial velocity standard 2MASS J0523-1403 (bottom). Spectra have been corrected for barycentric motion, normalized to the median flux and offset by integer constants. The $1.290 \mu \mathrm{m}$ Mn I line is labelled; other features arise primarily from $\mathrm{FeH}$ and $\mathrm{H}_{2} \mathrm{O}$ absorption.

Figures 5 through 7 display all of the reduced spectral data for SDSS J0006-0852 and LP 704-48 compared to select radial velocity standards. Signal-to-noise (S/N) for these data are generally $>50$, with the exception of our $\mathrm{N} 5$ data which has $\mathrm{S} / \mathrm{N} \approx 20$. All of the spectra show numerous molecular transitions arising from $\mathrm{H}_{2} \mathrm{O}$ and $\mathrm{FeH}$ in orders 59 and 49 , and $\mathrm{CO}$ in order 33 . We also detect the weak Mn I line at $1.2903 \mu \mathrm{m}$ in the spectrum of SDSS J0006-0852 (McLean et al. 2007).

\section{LP 704-48 AND SDSS J0006-0852}

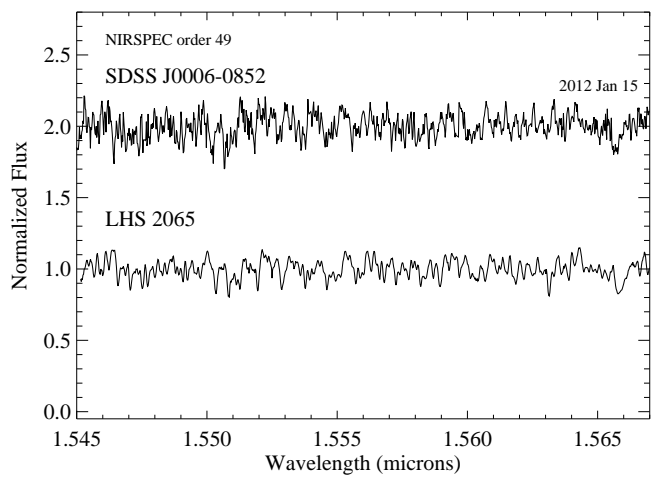

FIG. 6.- NIRSPEC order 49 (N5) spectrum of SDSS J0006-0852 obtained on 2012 Jan 15 (UT, top) compared to that of the M9 radial velocity standard LHS 2065 (bottom; from Prato et al. 2002). Spectra have been corrected for barycentric motion, normalized to the median flux and offset by integer constants. Features arise primarily from $\mathrm{H}_{2} \mathrm{O}$ and $\mathrm{FeH}$ absorption.

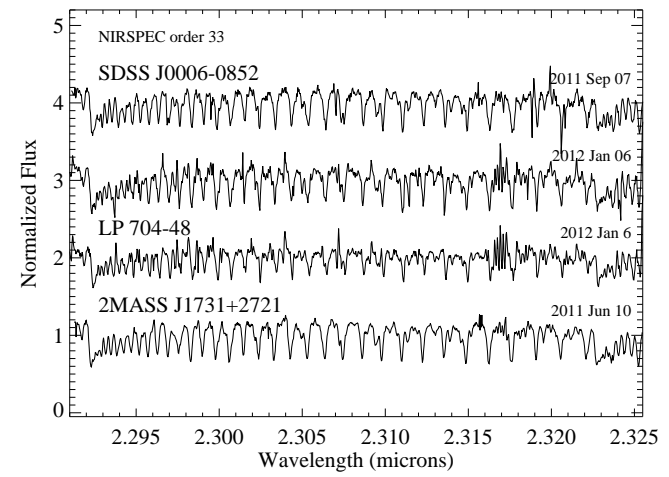

FIG. 7.- NIRSPEC order 33 (N7) spectra of SDSS J0006-0852 (top two spectra), LP 704-48 (second from the bottom) and the L0 radial velocity standard 2MASS J1731+2721 (bottom). Spectra have been corrected for barycentric motion, normalized to the median flux and offset by integer constants. Features arise primarily from $\mathrm{CO}$ absorption.

\subsection{Evidence for Common Proper Motion and Distance}

Our Nickel astrometry (Figure 21) shows convincingly that LP 704-48 and SDSS J0006-0852 comprise a common proper motion pair. To quantify this, we combined our measurements with prior astrometry from the SuperCosmos Sky Survey (Hambly et al. 2001c, b, a), the 2MASS All-Sky Point Source Catalog, and SDSS Data Release 7 (Abazajian et al. 2009). Linear fits yield proper motions of $\left[\mu_{\alpha} \cos \delta, \mu_{\delta}\right]=[-70 \pm 3,-314 \pm 2]$ mas $\mathrm{yr}^{-1}$ and $[-84 \pm 28,-337 \pm 17]$ mas $\mathrm{yr}^{-1}$ for LP $704-48$ and SDSS J0006-0852, respectively. The former agrees with values reported by Salim \& Gould (2003). These motions are consistent with each other to within $0.5 \sigma$ in Right Ascension and $1.3 \sigma$ in declination. The combined astrometry also yield angular separations of $25{ }^{\prime \prime} 26 \pm 0$ '. 09 in Right Ascension and $-10^{\prime \prime} 64 \pm 00^{\prime \prime} 17$ in declination, pointing from LP 704-48 to SDSS J0006-0852.

We also find that the estimated distances to these sources are in formal agreement, based on a combination of several optical and near-infrared abso-

\footnotetext{
${ }^{9}$ See http://www2.keck.hawaii.edu/inst/nirspec/redspec/index.h廿mpte magnitude/color and absolute magnitude/spectral
} 
TABLE 3

NIRSPEC OBSERVATIONS OF SDSS J0006-0852 AND LP 704-48

\begin{tabular}{|c|c|c|c|c|c|c|c|c|c|c|}
\hline Source & Date (UT) & MJD & Filter & $\lambda(\mu \mathrm{m})$ & Slit & Echelle/Grating $\left(^{\circ}\right)$ & $\mathrm{T}(\mathrm{s})$ & Airmass & Calibrator & Conditi \\
\hline SDSS J0006-0852 & 2010 Aug 19 & 55437.5 & N3 & $1.18-1.30$ & $0^{\prime \prime} 432 \times 12$ & $62.95 / 34.10$ & 3600 & $1.13-1.20$ & HD 3604 & clear, seeing $\approx$ \\
\hline SDSS J0006-0852 & 2010 Nov 26 & 55526.3 & N3 & $1.18-1.30$ & 0. & $62.95 / 34.10$ & 4000 & $1.14-1.33$ & HD 3604 & high humidity \\
\hline SDSS J0006-0852 & 2011 Jul 06 & 55748.6 & N3 & $1.18-1.30$ & $0 . \prime 432 \times 12$ & $62.95 / 34.08$ & 1500 & $1.14-1.16$ & HD 3604 & clear, seeing $\approx$ \\
\hline SDSS J0006-0852 & 2011 Sep 07 & 55811.5 & N7 & $2.00-2.39$ & $0 . .432 \times 12$ & $63.00 / 35.46$ & 2000 & $1.25-1.37$ & HD 13936 & light cirrus, se \\
\hline SDSS J0006-0852 & 2011 Sep 10 & 55814.5 & N3 & $1.18-1.30$ & 0 .' $432 \times 12$ & $62.95 / 34.08$ & 2000 & $1.24-1.41$ & HD 1154 & clear, seeing $\approx$ \\
\hline SDSS J0006-0852 & $2012 \operatorname{Jan} 6$ & 55932.2 & N7 & $2.00-2.39$ & $0 . .432 \times 12$ & $63.01 / 35.47$ & 2000 & $1.24-1.35$ & HD 1154 & clear, seeing $\approx$ \\
\hline LP $704-48$ & $2012 \operatorname{Jan} 6$ & 55932.2 & N7 & $2.00-2.39$ & $00^{\prime \prime} 432 \times 12$ & $63.01 / 35.47$ & 800 & $1.19-1.20$ & HD 1154 & clear, seeing $\approx($ \\
\hline SDSS J0006-0852 & 2012 Jan 15 & 55941.2 & N5 & $1.43-1.70$ & $0{ }^{\prime \prime} 288 \times 24$ & $63.04 / 36.30$ & 2400 & $1.28-1.40$ & 48 Cet & light clouds, see \\
\hline
\end{tabular}

TABLE 4

NiRSPEC Observations of Radial Velocity Calibrators

\begin{tabular}{|c|c|c|c|c|c|c|c|c|}
\hline Source & $\begin{array}{l}\text { Optical } \\
\text { SpT }\end{array}$ & $\begin{array}{l}\text { Obs. Date } \\
\text { (UT) }\end{array}$ & Filter & $\begin{array}{c}\text { Integration } \\
\text { (s) }\end{array}$ & Airmass & Calibrator & $\begin{array}{c}\mathrm{RV}^{\mathrm{a}} \\
\left(\mathrm{km} \mathrm{s}^{-1}\right)\end{array}$ & Refs \\
\hline 2MASS J05233822-1403022 & $\mathrm{L} 2.5$ & 2009 Oct 31 & N3 & 2000 & $1.20-1.22$ & $\tau$ Lep & $12.21 \pm 0.09$ & 1 \\
\hline 2MASS J09211410-2104446 & L1.5 & 2011 Oct 26 & N3 & 1200 & $1.32-1.33$ & HD 82724 & $80.53 \pm 0.11$ & 2 \\
\hline 2MASS J10220489+0200477 & L1 & 2011 Mar 18 & N7 & 1800 & 1.28 & 39 Uma & $19.29 \pm 0.11$ & 2 \\
\hline 2MASS J15150083+4847416 & L6 & 2011 Sep 10 & N7 & 350 & $1.57-1.63$ & HD 143187 & $-29.97 \pm 0.14$ & 3 \\
\hline DENIS-P J170548.38-051645.7 & $\mathrm{L} 4$ & 2011 Aug 11 & N7 & 1200 & 1.24 & HD 159415 & $12.19 \pm 0.11$ & 4 \\
\hline 2MASS J17312974+2721233 & LO & 2011 Jun 10 & N7 & 1000 & 1.03 & HD 165029 & $-29.76 \pm 0.11$ & 2 \\
\hline 2MASS J22244381-0158521 & L4.5 & 2011 Sep 07 & N7 & 1500 & $1.08-1.11$ & HD 198070 & $-37.55 \pm 0.09$ & 5 \\
\hline
\end{tabular}

References. - (1) Cruz et al. (2003); (2) Reid et al. (2008); (3) Wilson et al. (2003); (4) Kendall et al. (2004); (5) Kirkpatrick et al. $(2000)$.

${ }^{a}$ Radial velocity measurements from Blake et al. (2010).

TABLE 5

Distance Estimates FOR SDSS J0006-0852 AND LP $704-48$

\begin{tabular}{|c|c|c|c|}
\hline \multirow[b]{2}{*}{ Method } & \multicolumn{3}{|c|}{ Distance $(\mathrm{pc})$} \\
\hline & LP 704-48 & SDSS J0006-0852 & $\Delta / \sigma$ \\
\hline H02 $M_{i}$ vs $i-z$ & $28 \pm 5$ & $31 \pm 6$ & -0.4 \\
\hline H02 $M_{i}$ vs $i-J$ & $31 \pm 6$ & $30 \pm 6$ & +0.1 \\
\hline $\mathrm{H} 02 M_{J}$ vs SpT & $17 \pm 4$ & $36 \pm 7$ & -2.4 \\
\hline $\mathrm{C} 03 M_{J}$ vs SpT & $18 \pm 3$ & $35 \pm 4$ & -3.4 \\
\hline W05 $M_{i}$ vs $i-z$ & $17 \pm 2$ & $33 \pm 5$ & -3.0 \\
\hline W05 $M_{i}$ vs $i-J$ & $27 \pm 3$ & $31 \pm 3$ & -0.3 \\
\hline $\mathrm{B} 10 M_{r}$ vs $r-z$ & $22 \pm 4$ & $\ldots b$ & $\ldots$ \\
\hline $\mathrm{B} 10 M_{r}$ vs $r-i$ & $21 \pm 4$ & $\ldots b$ & $\cdots$ \\
\hline $\mathrm{B} 10 M_{r}$ vs $i-z$ & $26 \pm 6$ & $\ldots \mathrm{b}$ & $\cdots$ \\
\hline S10 $M_{i}$ vs $i-z$ & $\ldots b$ & $31 \pm 8$ & $\ldots$ \\
\hline $\mathrm{S} 10 M_{i}$ vs $i-J$ & $\ldots \mathrm{b}$ & $36 \pm 6$ & $\ldots$ \\
\hline $\mathrm{D} 12 M_{J}$ vs $\mathrm{SpT}^{\mathrm{a}}$ & $20 \pm 5$ & $37 \pm 8$ & -1.8 \\
\hline $\mathrm{D} 12 M_{H}$ vs SpT ${ }^{\mathrm{a}}$ & $20 \pm 4$ & $40 \pm 8$ & -2.2 \\
\hline D12 $M_{K}$ vs SpTa & $20 \pm 4$ & $42 \pm 9$ & -2.2 \\
\hline Weighted Mean & $22 \pm 5$ & $32 \pm 2$ & -2.0 \\
\hline
\end{tabular}

REFERENCES. - (H02): Hawley et al. (2002) ; (C03): Cruz et al. (2003) ; (W05): West et al. (2005); (B10): Bochanski et al. (2010); (S10): Schmidt et al. (2010); (D12): Dupuv \& Liu 2012a

a Excluded from final average.

b Outside defined color range.

type relationship: 10 defined in Hawlev et al. (2002); Cruz et al. (2003); West et al. (2005); Schmidt et al. (2010); Bochanski et al. (2010); and Dupuy \& Liu (2012a). Table 5 summarizes these estimates, which in-

10 Note that linear coefficient of $M_{r}$ versus $r-i$ in Table 4 of Bochanski et al. (2010) should be +4.548 , not -4.548 . corporate uncertainties in photometry, spectral classifications ( 0.5 subtypes), and the reported systematic uncertainty in the absolute magnitude relations. Some relationships show better agreement between the two components than others, with the absolute magnitude/spectral type relations diverging the most. Using only the color relations, we find mean distances of $22 \pm 5$ pc for LP 70448 and $32 \pm 2$ pc for SDSS J0006-0852, a $2 \sigma$ difference. Note that nearly all of the relations place LP 704-48 roughly $50 \%$ closer, suggesting that it to may be an unresolved binary, although age/surface gravity effects cannot be ruled out (see below). We adopt a weighted mean of $30 \pm 3 \mathrm{pc}$ for the combined system, implying a projected separation of $820 \pm 120 \mathrm{AU}$.

\subsection{Probability of Association}

To assess the probability of chance alignment for this wide pairing, we followed the method described in Dhital et al. (2010), which estimates the frequency of unrelated pairings using a Galactic model based on an empirical luminosity function (Jurić et al. 2008; Bochanski et al. 2010) and an empirical space velocity distribution (Bochanski et al. 2007a). The number of single (and hence unrelated) stars within a 6D ellipsoid defined by the angular separation of the binary, the estimated distance to the binary, the space motions of the binary, and the uncertainties in these values was determined through Monte Carlo simulation. From $10^{6}$ simulations, we found that only 0.0015 stars were spatially coincident and had proper motions similar to the values observed for this wide pair. When the (far more precise) radial motions of SDSS J0006-0852 and LP 704-48 were 
also considered (see below), the number of chance alignments fell to zero (i.e. probability $<10^{-6}$ ). We therefore conclude with high confidence that LP 704-48 and SDSS J0006-0852 are not a chance alignment.

\subsection{Activity, Age and Metallicity}

Additional constraints on the physical properties of this system can be inferred from our optical spectroscopy of LP 704-48 and SDSS J0006-0852. The lack of Li I absorption in either source rules out masses below $0.06 \mathrm{M}_{\odot}$, implying ages older than $90 \mathrm{Myr}$ and $210 \mathrm{Myr}$, respectively, based on the evolutionary models of Baraffe et al. (2003) and $\mathrm{T}_{\text {eff }}$ estimates of $2660 \pm 150 \mathrm{~K}$ and $2400 \pm 160 \mathrm{~K}$ as inferred from the $\mathrm{T}_{\text {eff }} / \mathrm{SpT}$ relation of Stephens et al. (2009) $\square$ More stringent age constraints come from the lack of $\mathrm{H} \alpha$ emission in either source. For SDSS J0006-0852, one could attribute the lack of emission to an increasingly neutral photosphere that is decoupled from the magnetic field, a hypothesis used to explain the decline in both $\mathrm{H} \alpha$ and X-ray emission in dwarfs later than M8-M9 (e.g. Gizis et al. 2000b; Mohanty et al. 2002; West et al. 2004). However, the inactivity of LP 704-48 is remarkable given the high incidence of $\mathrm{H} \alpha$ emission among M7 dwarfs in the vicinity of the Sun $(\gtrsim 90 \%$ of sources with vertical scaleheights $|Z|<50$ pc; Hawlev et al. 1996; Gizis et al. 2000b; West et al. 2004, 2006, 2008). From the activity frequencies of $\mathrm{M}$ dwarfs at various scaleheights, West et al. (2008) have inferred an activity lifetime for M7 dwarfs of $8_{-1.0}^{+0.5}$ Gyr. Since emission from LP 704-48 is seen in neither our spectrum nor that of Cruz \& Reid (2002), and given that the limit on $\mathrm{H} \alpha$ luminosity is nearly two orders of magnitude below the local active mean $\left(\log _{10} L_{H \alpha} / L_{b o l}=-4.3\right.$; West et al. 2004), we conclude that this source is truly inactive and that the LP 704-48/SDSS J0006-0852 system is likely to be quite old.

This conclusion is supported by the kinematics of the system. Combining our proper motion measurement, distance estimate and systemic radial velocity determination below, we derive the heliocentric $U V W$ velocities listed in Table 6. These velocities are on the boundary of the young-old disk as defined in Leggett (1992). The disk classification is consistent with what appear to be nearsolar metallcities for LP 704-48 and SDSS J0006-0852, as evident from their spectral energy distributions and as quantified by the $\zeta$ index of Lépine et al. (2007). Both sources have $\zeta \approx 1.00$ (Table 2) indicating roughly solar metallicities.

We therefore conclude that LP 704-48 and SDSS J0006-0852 comprise a physically associated, widely-separated system of relatively old, VLM stars with near-solar metallicity.

\section{THE M DWARF/T DWARF SPECTRAL BINARY} SDSS J0006-0852

As discussed in Section 2.1, the peculiar features observed in the low-resolution near-infrared spectrum of SDSS J0006-0852 indicate the presence of an unseen $\mathrm{T}$ dwarf companion. To test this hypothesis, we performed a spectral template fitting analysis similar to

11 Uncertainties include classification of 0.5 subtypes for both components and a $100 \mathrm{~K}$ systematic uncertainty in the Stephens et al. (2009) relation. that described in Burgasser et al. (2010). We drew 638 spectra of 614 sources with $\mathrm{S} / \mathrm{N} \gtrsim 20$ from the SpeX Prism Spectral Librarie: 12 with published optical and/or near-infrared spectral types between M7 and T8, excluding known binaries, subdwarfs and low-surface gravity brown dwarfs. The spectra were reclassified in the near-infrared using the index-based scheme defined in Burgasser (2007a). Spectral fluxes were then scaled to absolute $F_{\lambda}$ units using the $M_{J} /$ spectral type relation of Cruz et al. (2003) for types M7-L2 and the $M_{K_{s}}$ /spectral type relation of Looper et al. (2008a) for types L2-T8. We then combined pairs of flux-calibrated spectra to create binary spectral templates $(B(\lambda))$, constraining the spectral types of the primary to M7-L8 and of the secondary to L9-T8, thereby generating 44605 unique binary templates. We compared both the original 638 single spectra and the binary templates to the spectrum of SDSS J0006-0852 $(S(\lambda))$ over the wavelength ranges $\{\lambda\}=0.95-1.35 \mu \mathrm{m}, 1.45-1.80 \mu \mathrm{m}$ and $2.00-2.35 \mu \mathrm{m}$ using the $\chi^{2}$ statistic,

$$
\chi^{2}=\sum_{\{\lambda\}}\left(\frac{B(\lambda)-S(\lambda)}{\sigma(\lambda)}\right)^{2}
$$

where $\sigma(\lambda)$ corresponds to the uncertainty spectrum of SDSS J0006-0852 alone.

Figure 8 displays the four best-fitting binary templates (the best-fitting single template, HB 2115-4518, is shown in Figure 4). A combination of a late-type $\mathrm{M}$ dwarf and mid-type $\mathrm{T}$ dwarf accurately reproduces the spectrum of SDSS J0006-0852, including the excess flux at $1.27 \mu \mathrm{m}, 1.55 \mu \mathrm{m}$ and $2.1 \mu \mathrm{m}$. This combination also reproduces with reasonable fidelity the notch feature at $1.62 \mu \mathrm{m}$, arising from $\mathrm{FeH}$ absorption in the $\mathrm{M}$ dwarf primary and the pseudo-continuum peak blueward of the $1.6 \mu \mathrm{m} \mathrm{CH}_{4}$ band in the $\mathrm{T}$ dwarf secondary (Burgasser $2007 \mathrm{~b})$. Several dozen binary templates were found to provide statistically superior matches to the spectrum of SDSS J0006-0852 as compared to HB 2115-4518 alone $\left(\chi^{2}=12.5\right)$, with significance values $>99 \%$ based on the F-test statistic (see Eqns. 2-6 in Burgasser et al. 2010). Table 7 details the ten best binary template fits, including component names, near-infrared spectral types and relative $J H K$ magnitudes on the MKO [3 photometric system. Averaging over all fits with an F-test statistical weighting, we infer mean component types of M8.5 \pm 0.5 and $\mathrm{T} 5 \pm 1$, both rounded off to the nearest half-subclass. The near-infrared classification of the primary is consistent with the combined-light optical classification. We also estimate relative magnitudes of $3.2 \pm 0.3,3.8 \pm 0.5$ and $4.4 \pm 0.6$ in the $J-, H$ - and $K$-bands. The component properties of SDSS J0006-0852 are essentially identical to those inferred for 2MASS J0320-0446AB by Burgasser et al. (2008a), which is not unexpected given

12 See http://www.browndwarfs.org/spexprism Data were drawn from Burgasser et al. (2004, 2006a,b, 2007a, 2008a b, 2010); Cruz et al. (2004); Burgasser \& McElwain (2006); Chiu et al. (2006); McElwain \& Burgasser (2006); Reid et al. (2006); Burgasser (2007a|b|c); Liebert \& Burgasser (2007); Looper et al. (2007, 2008b); Luhman et al. (2007); Siegler et al. (2007); Sheppard \& Cushing (2009); Kirkpatrick et al. (2010); Cruz et al. in prep.; and Burgasser et al. in prep.

${ }^{13}$ Mauna Kea Observatory filter set; see Tokunaga et al. (2002) and Simons \& Tokunaga (2002). 
TABLE 6

Properties of the LP 704-48/SDSS J0006-0852AB System

\begin{tabular}{|c|c|c|c|c|}
\hline Parameter & LP 704-48 & SDSS J0006-0852AB & System & Ref. \\
\hline Optical SpT & M7 & M9 & $\ldots$ & $1,2,3$ \\
\hline NIR SpT & $\ldots$ & $\mathrm{M} 8.5 \pm 0.5+\mathrm{T} 5 \pm 1$ & $\ldots$ & 1 \\
\hline$r$ & $17.313 \pm 0.005$ & $21.04 \pm 0.06$ & $\ldots$ & 4 \\
\hline$r-i$ & $2.373 \pm 0.006$ & $2.82 \pm 0.06$ & $\ldots$ & 4 \\
\hline$i-z$ & $1.197 \pm 0.006$ & $1.77 \pm 0.03$ & $\ldots$ & 4 \\
\hline$i-J$ & $1.78 \pm 0.02$ & $2.32 \pm 0.04$ & $\ldots$ & 4,5 \\
\hline$J$ & $11.97 \pm 0.02$ & $14.14 \pm 0.04$ & $\ldots$ & 5 \\
\hline$J-K_{s}$ & $0.88 \pm 0.03$ & $1.01 \pm 0.05$ & $\ldots$ & 5 \\
\hline $\log _{10} L_{H \alpha} / L_{b o l}$ & $<-6.2$ & $<-5.7$ & $\ldots$ & 1 \\
\hline Est. $d(\mathrm{pc})$ & $22 \pm 5$ & $32 \pm 2$ & $30 \pm 3$ & 1 \\
\hline$\mu_{\alpha}\left(\operatorname{mas~yr}^{-1}\right)$ & $-70 \pm 3$ & $-84 \pm 28$ & $-70 \pm 3$ & 1 \\
\hline$\mu_{\delta}\left({\left.\operatorname{mas~} \mathrm{yr}^{-1}\right)}^{\prime}\right.$ & $-314 \pm 2$ & $-337 \pm 17$ & $-315 \pm 2$ & 1 \\
\hline $\mathrm{RV}\left(\mathrm{km} \mathrm{s}^{-1}\right)$ & $-15.3 \pm 0.9$ & Variable & $-15.3 \pm 0.3^{\mathrm{a}}$ & 1 \\
\hline$a(\mathrm{AU})$ & $\ldots$ & $0.286 \pm 0.009$ & $820 \pm 120$ & 1 \\
\hline$a\left({ }^{\prime \prime}\right)$ & $\ldots$ & $0 .{ }^{\prime \prime} 0095 \pm 0 . \prime 0010^{\mathrm{b}}$ & $27^{\prime \prime} 41 \pm 0 . \prime 10$ & 1 \\
\hline$U\left(\mathrm{~km} \mathrm{~s}^{-1}\right)$ & $\ldots$ & $\ldots$ & $31 \pm 3$ & 1 \\
\hline$V\left(\mathrm{~km} \mathrm{~s}^{-1}\right)$ & $\ldots$ & $\ldots$ & $-37 \pm 3$ & 1 \\
\hline$W\left(\mathrm{~km} \mathrm{~s}^{-1}\right)$ & $\ldots$ & $\ldots$ & $2 \pm 2$ & 1 \\
\hline Est. Age (Gyr) & $\gtrsim 8$ & $\gtrsim 3-4$ & 邓3-4 & 1,2 \\
\hline Est. Masses $\left(\mathrm{M}_{\odot}\right)^{\mathrm{c}}$ & 0.092 & $0.082-0.083,0.049-0.064$ & $0 . \widetilde{22}-0.24$ & 1 \\
\hline
\end{tabular}

ReFerences. - (1) This paper; (2) West et al. (2008); (3) Cruz \& Reid (2002); (4) SDSS:

York et al. (2000); (5) 2MASS: Skrutskie et al. (2006).

a Based on constrained radial velocity orbit fit to SDSS J0006-0852AB; see Table 10

b At maximum elongation.

c For an age range of 3-10 Gyr, based on the evolutionary models of Baraffe et al. (2003).

TABLE 7

Best-Fitting Spectral Binary Templates for SDSS J0006-0852.

\begin{tabular}{|c|c|c|c|c|c|c|c|}
\hline Primary & $\mathrm{SpT}$ & Secondary & $\mathrm{SpT}$ & $\Delta J_{M K O}$ & $\Delta H_{M K O}$ & $\Delta K_{M K O}$ & $\chi^{2}$ \\
\hline HB 2115-4518 & M8.6 & 2MASS J21513839-4853542 & $\mathrm{T} 4.2$ & 2.886 & 3.384 & 3.908 & 5.13 \\
\hline DENIS-P J2353-0833 & M8.6 & SDSS J135852.68+374711.9 & $\mathrm{T} 4.8$ & 2.930 & 2.667 & 3.471 & 5.16 \\
\hline HB $2115-4518$ & M8.6 & SDSS J074149.15+235127.5 & $\mathrm{T} 5.4$ & 3.096 & 3.880 & 4.457 & 5.31 \\
\hline HB $2115-4518$ & M8.6 & SDSS J083048.80+012831.1 & $\mathrm{T} 4.9$ & 2.953 & 3.598 & 4.168 & 5.33 \\
\hline HB 2115-4518 & M8.6 & 2MASSI J2254188+312349 & T3.9 & 3.097 & 3.455 & 3.923 & 5.50 \\
\hline DENIS-P J2353-0833 & M8.6 & 2MASSI J0937347+293142 & T5.6 & 3.199 & 3.086 & 3.981 & 5.53 \\
\hline DENIS-P J2353-0833 & M8.6 & SDSS J083048.80+012831.1 & T4.9 & 3.285 & 2.950 & 3.607 & 5.54 \\
\hline HB 2115-4518 & M8.6 & SDSS J074201.41+205520.5 & $\mathrm{T} 5.2$ & 3.107 & 3.838 & 4.492 & 5.54 \\
\hline HB 2115-4518 & M8.6 & 2MASS J05591914-1404488 & $\mathrm{T} 4.4$ & 3.019 & 3.615 & 4.176 & 5.56 \\
\hline НВ 2115-4518 & M8.6 & 2MASS J06020638+4043588 & $\mathrm{T} 4.6$ & 2.986 & 3.637 & 4.200 & 5.57 \\
\hline Average Primary & $\mathrm{M} 8.7 \pm 0.2$ & Average Secondary & $\mathrm{T} 4.8 \pm 1.1$ & $3.2 \pm 0.3$ & $3.8 \pm 0.5$ & $4.4 \pm 0.6$ & $99.8 \%^{a}$ \\
\hline
\end{tabular}

a Confidence level that the best-fit binary template provides a statistically better match than the best-fit single template (HB $2115-4518, \chi^{2}=12.6$ ) based on the F-test statistic.

the similarity in their near-infrared spectra (Figure 4).

\section{RADIAL VELOCITY VARIABILITY AND ORBIT}

\subsection{Radial Velocity Measurements}

While our spectral template analysis provides compelling evidence that SDSS J0006-0852 harbors a T dwarf companion, we sought independent verification by searching for radial velocity variations in the NIRSPEC data. Velocities were determined by cross-correlating the SDSS J0006-0852 and LP 704-48 spectra with each of the radial velocity templates observed in the same order using the IDL xcorl package (Mohanty \& Basri 2003; West \& Basri 2009). We correlated the spectra over the wavelength ranges $1.283-1.299 \mu \mathrm{m}$ (order 59), 1.5455-1.5665 $\mu \mathrm{m}$ (order 49) and 2.297-2.325 $\mu \mathrm{m}$ (order 33), in each case sampling ten independent windows equally-spaced in logarithmic wavelength. The individual correlations within a given order were combined using an outlier-resistant mean based on comparison of median and median absolute deviation statistics (with a $2.5 \sigma$ threshhold), and velocities determined from multiple standards in a given epoch (Table ??) were combined using an uncertainty-weighted mean. We also measured the radial velocities for each of the standards in order to assess systematic effects. We found these measurements, with uncertainties ranging from $0.5-0.9 \mathrm{~km} \mathrm{~s}^{-1}$, were all consistent, albeit with lower precision, with those reported in Blake et al. (2010) to within 1.6 $\sigma$. While a forward-modeling approach would have likely produced better precision, our uncertainties are nevertheless roughly equivalent to contemporary NIRSPEC RV measurements of very cool dwarfs (e.g., Prato et al. 2002; Zapatero Osorio et al. 2007; Blake et al. 2008, 2010; Rice et al. 2010; Rodler et al. 2012).

Table ?? lists the final radial velocity measurements for SDSS J0006-0852 for each epoch. There is clear varia- 
TABLE 8

Radial Velocity Measurements of SDSS J0006-0852 and LP 704-48 By Comparator (in Km S ${ }^{-1}$ )

\begin{tabular}{|c|c|c|c|c|c|c|c|c|}
\hline MJD & Order & $\begin{array}{c}\mathrm{J} 0921-2104 \\
(80.53 \pm 0.11)\end{array}$ & $\begin{array}{c}\text { J0523-1403 } \\
(12.21 \pm 0.09)\end{array}$ & $\begin{array}{c}\mathrm{J} 1515+4849 \\
(-29.97 \pm 0.14)\end{array}$ & $\begin{array}{c}\mathrm{J} 1731+2721 \\
(-29.76 \pm 0.11)\end{array}$ & $\begin{array}{c}\mathrm{J} 2224-0158 \\
(-37.55 \pm 0.09)\end{array}$ & $\begin{array}{c}\mathrm{J} 1022+5825 \\
(19.29 \pm 0.11)\end{array}$ & $\begin{array}{c}\mathrm{J} 1705-0516 \\
(12.19 \pm 0.11)\end{array}$ \\
\hline & & \multicolumn{7}{|c|}{ SDSS J0006-0852 } \\
\hline 55427.53279 & 59 & $-7.36 \pm 0.38$ & $-7.08 \pm 0.46$ & & & & & \\
\hline 55526.27187 & 59 & $-18.39 \pm 0.50$ & $-17.99 \pm 0.36$ & $\ldots$ & $\ldots$ & $\ldots$ & $\cdots$ & $\cdots$ \\
\hline 55748.61963 & 59 & $-15.57 \pm 0.93$ & $-15.4 \pm 1.5$ & $\ldots$ & $\ldots$ & $\ldots$ & $\ldots$ & \\
\hline 55811.54273 & 33 & & & $-20.90 \pm 0.65$ & $-20.22 \pm 0.28$ & $-21.1 \pm 1.1$ & $-22.17 \pm 0.38$ & $-22.8 \pm 1.1$ \\
\hline 55814.53006 & 59 & $-20.17 \pm 0.74$ & $-19.07 \pm 0.85$ & & & & & \\
\hline 55932.23208 & 33 & $\ldots$ & $\ldots$ & $-22.60 \pm 0.77$ & $-22.89 \pm 0.45$ & $-23.3 \pm 1.4$ & $-24.32 \pm 0.51$ & $-25.35 \pm 0.45$ \\
\hline 55941.19343 & 49 & $\ldots$ & $\ldots$ & $\ldots$ & $\ldots$ & $\ldots$ & $\ldots$ & $\ldots$ \\
\hline & & & & & LP 704-48 & & & \\
\hline
\end{tabular}

Note. - Radial velocities (RVs) are given in the heliocentric reference frame for the observed Modified Julian Date (MJD; Julian Date - 2400000).

TABLE 9

Radial Velocity Measurements for SDSS J0006-0852

\begin{tabular}{lcccc}
\hline \hline Date (UT) & MJD & Order & $\begin{array}{c}\text { RV } \\
\left(\mathrm{km} \mathrm{s}^{-1}\right)\end{array}$ & $\begin{array}{c}\sigma \\
\left(\mathrm{km} \mathrm{s}^{-1}\right)\end{array}$ \\
& & & & \\
\hline 2010 Aug 19 & 55427.53279 & 59 & -7.25 & 0.30 \\
2010 Nov 26 & 55526.27187 & 59 & -18.13 & 0.30 \\
2011 Jul 06 & 55748.61963 & 59 & -15.51 & 0.78 \\
2011 Sep 07 & 55811.54273 & 33 & -20.98 & 0.92 \\
2011 Sep 10 & 55814.53006 & 59 & -19.70 & 0.56 \\
2012 Jan 06 & 55932.23208 & 33 & -24.0 & 1.1 \\
2012 Jan 15 & 55941.19343 & 49 & -21.6 & 1.3 \\
\hline
\end{tabular}

Note. - Radial velocities (RVs) are given in the heliocentric reference frame for the observed Modified Julian Date (MJD; Julian Date - 2400000).

tion in the velocities, with values ranging from -24 to $-7 \mathrm{~km} \mathrm{~s}^{-1}$ over the 16 months of observation. These values straddle the radial velocity measured for LP 704-48, $-15.6 \pm 0.4 \mathrm{~km} \mathrm{~s}^{-1}$, which we consider to be an estimate of the systemic radial velocity (see below). The $\chi^{2}$ for these data, 1000 with 6 degrees of freedom, rules out a constant radial motion, indicating the presence of a gravitationally perturbing companion.

\subsection{Radial Velocity Orbit: MCMC Analysis}

With seven measurements in hand, we have a minimum set necessary to constrain a radial velocity orbit for SDSS J0006-0852. To do this, we performed a Monte Carlo Markov Chain (MCMC) analysis following Ford (2005). We defined the parameter vector:

$$
\vec{\theta}=\left\{\ln P, \ln K_{1}, \sqrt{e} \cos \omega, \sqrt{e} \sin \omega, \phi_{1}, V_{c o m}\right\},
$$

where $P$ is the period of the orbit in years, $K_{1}$ the semiamplitude of the primary's orbital motion along the line of sight, $e$ the eccentricity, $\omega$ the argument of periastron, $\phi_{1}$ the mean anomaly in the first epoch $\left(T_{1}\right)$ of data, and $V_{\text {com }}$ the system's center of mass radial velocity. Note that the epoch of periastron passage is $T_{0}=T_{1}-P \phi_{1} / 2 \pi$. As discussed in Ford (2005), this particular configuration of orbital parameters is found to improve convergence for MCMC analysis in cases of small $e$. The semi-amplitude, period and eccentricity are related to the mass function:

$$
f_{M} \sin i \equiv \frac{\mathrm{M}_{2} \sin i}{\left(\mathrm{M}_{1}+\mathrm{M}_{2}\right)^{2 / 3}}=K_{1}\left(\frac{P}{2 \pi G}\right)^{1 / 3} \sqrt{1-e^{2}}
$$

where $i$ is the inclination of the orbital plane with respect to the plane of the sky $\left(0^{\circ}\right.$ corresponds to a face-on projection), and $M_{1}$ and $M_{2}$ are the masses of the $M$ and T dwarf components of SDSS J0006-0852, respectively. We performed three sets of $300 \mathrm{MCMC}$ trials to maximally explore the six-dimensional parameter space. For each trial, we started with an initial parameter set $\overrightarrow{\theta_{0}}$ selected from distributions derived from the observational data. For $P$ and $K_{1}$, we drew from Gaussian distributions with means of $0.5 \mathrm{yr}$ and $9 \mathrm{~km} \mathrm{~s}^{-1}$ and widths $0.2 \mathrm{yr}$ and $3 \mathrm{~km} \mathrm{~s}^{-1}$, derived from the scales over which the radial velocities reversed in trend. For $e, \omega$ and $\phi_{1}$, we drew from uniform distributions spanning ranges $[0,0.9],[0,2 \pi]$ and $[0,2 \pi]$, respectively. For $V_{\text {com }}$, we drew from a Gaussian distribution centered on the observed radial velocity of LP 704-48 with a standard deviation of $0.6 \mathrm{~km} \mathrm{~s}^{-1}$, under the assumption that the relative orbital motion of the pair is negligible $\left(\lesssim 0.5 \mathrm{~km} \mathrm{~s}^{-1}\right)$ Throughout the simulation, we imposed boundary conditions on the parameters of $0.05 \lesssim P \lesssim 10 \mathrm{yr}, 1 \lesssim K_{1} \lesssim 100 \mathrm{~km} \mathrm{~s}^{-1}$ and $e \leq 0.95$.

For each step in the MCMC chain, we computed a new vector $\overrightarrow{\theta^{\prime}}$ by changing one parameter $\theta_{j}$, drawing from a Gaussian distribution

$$
q\left(\theta_{j}^{\prime} \mid \theta_{j}\right) \propto e^{-\frac{\left(\theta_{j}^{\prime}-\theta_{j}\right)^{2}}{2 \beta_{j}}}
$$

where $\vec{\beta}$ is a vector of scale factors. Initial scale factors were chosen by trial and error to optimize acceptance rates (Gelman \& Rubin 1992). We also found it useful to allow these scale factors to increase by $20 \%$ (up to a factor of 20) every 500 chain steps after the associated parameter was last updated. With each parameter set, we computed that model's radial velocities $\left(V_{\text {mod }}\right)$ at the epochs of observation and the $\chi^{2}$ deviation with the measured values $\left(V_{o b s}\right)$ :

$\chi^{2}=\sum_{k}\left(\frac{V_{m o d, k}-V_{o b s, k}}{\sigma_{o b s, k}}\right)^{2}+\left(\frac{V_{c o m}-V_{L P} 704-48}{\sigma_{L P} 704-48}\right)^{2}$, 

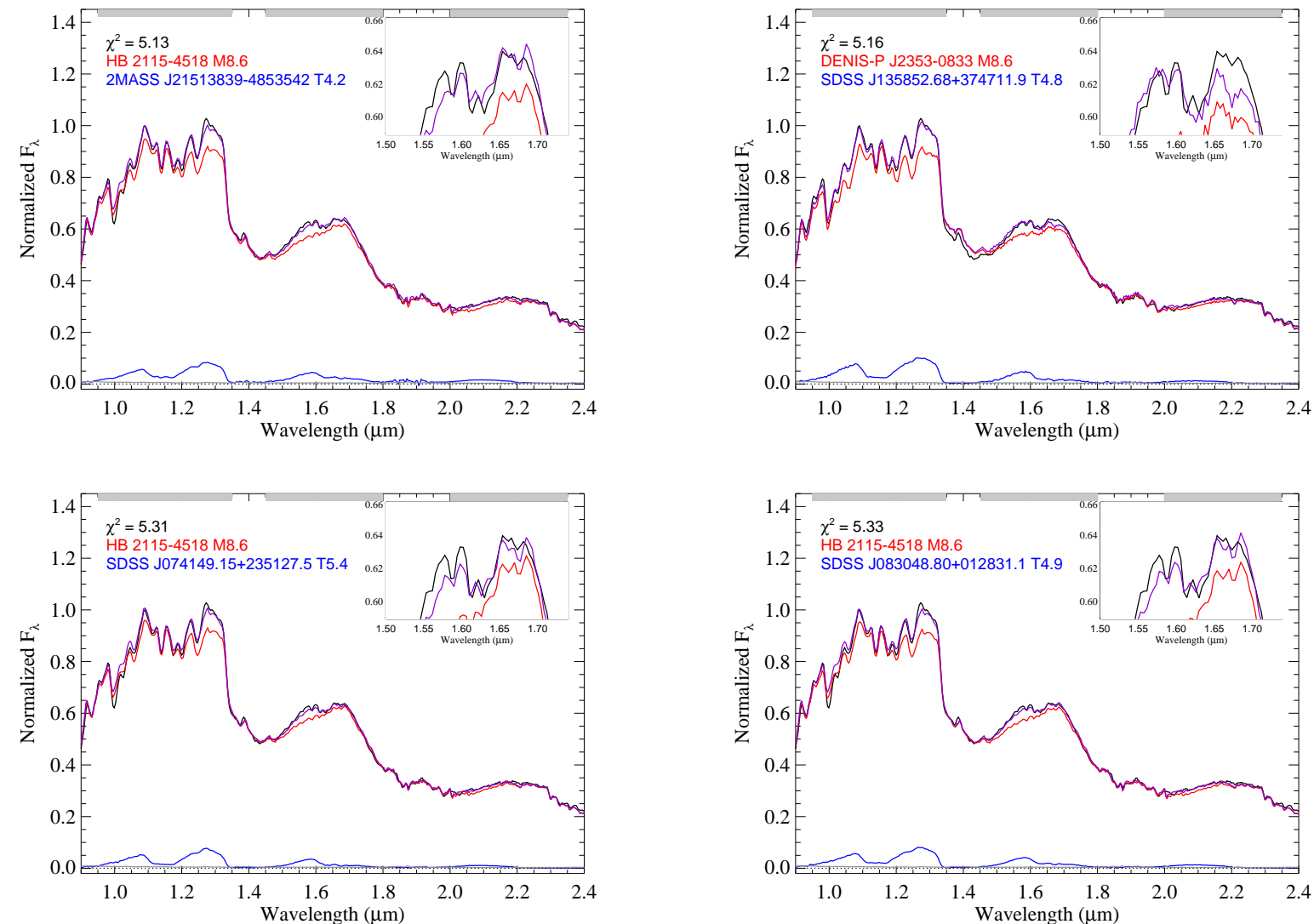

FIG. 8. - SpeX spectrum of SDSS J0006-0852 (black lines) compared to the four best-fitting binary spectral templates (purple lines), all normalized in the 1.1-1.3 $\mu \mathrm{m}$ region. Primary (red lines) and secondary (blue lines) component spectra for the spectral binaries are also shown, scaled according to their contribution to the combined-light spectrum (source names in each panel are listed). The uncertainty spectrum of SDSS J0006-0852 is indicated as the grey line at bottom. Shaded regions at the top of the panels indicate the spectral ranges over which the spectrum of SDSS J0006-0852 and the binary templates were compared, and $\chi^{2}$ values are listed. The inset boxes show a close up of the 1.50-1.75 $\mu \mathrm{m}$ region where the notch feature is detected. 


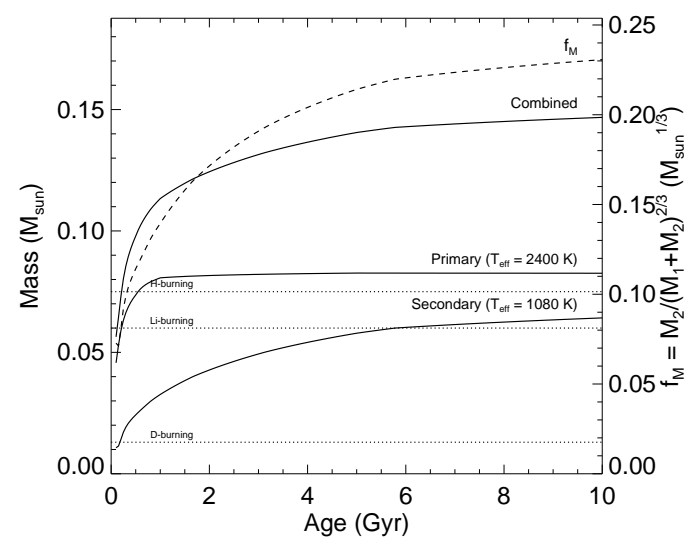

Fig. 9.- Expected component and combined masses of SDSS J0006-0852AB (solid black curves) as a function of age based on the evolutionary models of Baraffe et al. (2003) and assuming component $\mathrm{T}_{e f f} \mathrm{~s}$ of $2400 \mathrm{~K}$ and $1080 \mathrm{~K}$ for the M8.5 primary and $\mathrm{T} 5$ secondary, respectively. The system mass function, $f_{\mathrm{M}}=\mathrm{M}_{2} /\left(\mathrm{M}_{1}+\mathrm{M}_{2}\right)^{2 / 3}$ (dashed line) is also shown, with values corresponding to the right axis. Hydrogen-, lithium- and deuterium-burning mass limits are indicated by the horizontal dotted lines.

where the sum is over all observations. Note that we have explicitly included the measurement of the radial motion of LP 704-48 as an "observation" of the systemic motion for SDSS J0006-0852AB. The new parameter set $\overrightarrow{\theta^{\prime}}$ replaced $\vec{\theta}$ if

$$
U(0,1) \leq e^{\left(\chi^{2 \prime}-\chi^{2}\right) / 2},
$$

where $U(0,1)$ is a random number drawn from a uniform distribution between 0 and 1 . This process was repeated cyclically for each parameter 100,000 times for each of the 300 trials.

While these data are nominally sufficient to constrain the radial velocity orbit of the system, we also incorporated constraints based on the physical properties of the components as inferred from their spectral types and evolutionary models. Using the Stephens et al. (2009) $\mathrm{T}_{\text {eff }} / \mathrm{SpT}$ relation, we adopted component effective temperatures of $2400 \pm 160 \mathrm{~K}$ and $1080 \pm 170 \mathrm{~K}$ for SDSS J0006-0852A and B, respectively, and used these to estimate component masses as a function of age with the evolutionary models of Baraffe et al. (2003). Figure 9 plots these masses, the combined system mass and the mass function (Eqn. 3) for ages of 0.1-10 Gyr. Note that the estimated mass of SDSS J0006-0852A asymptotes to $0.082 \mathrm{M}_{\odot}$ beyond 2 Gyr, while the estimated mass of SDSS J0006-0852B (and by extension the combined mass and mass ratio) increase monotonically from $0.033 \mathrm{M}_{\odot}$ to $0.064 \mathrm{M}_{\odot}$ for ages 1-10 Gyr, never breaching the minimum mass for hydrogen burning. The mass ratio $\left(q \equiv \mathrm{M}_{2} / \mathrm{M}_{1}\right)$ ranges from 0.41 at $1 \mathrm{Gyr}$ to 0.78 at 10 Gyr. The evolutionary estimates of $f_{M}$ constrain the allowed orbital solutions by the requirement that

$$
f_{M}^{o r b i t} \sin i \leq \operatorname{maximum}\left(f_{M}^{\text {model }}\right) \approx 0.23 \mathrm{M}_{\odot}^{1 / 3}
$$

(i.e., $\sin i \leq 1$ ). To explore the systematic effects of our external constraints on the orbit model, we performed separate MCMC trials with both $V_{C O M}$ and evolutionary model constraints (Case A), without the $V_{C O M}$ constraints (Case B) and with neither the $V_{C O M}$ nor evolu-
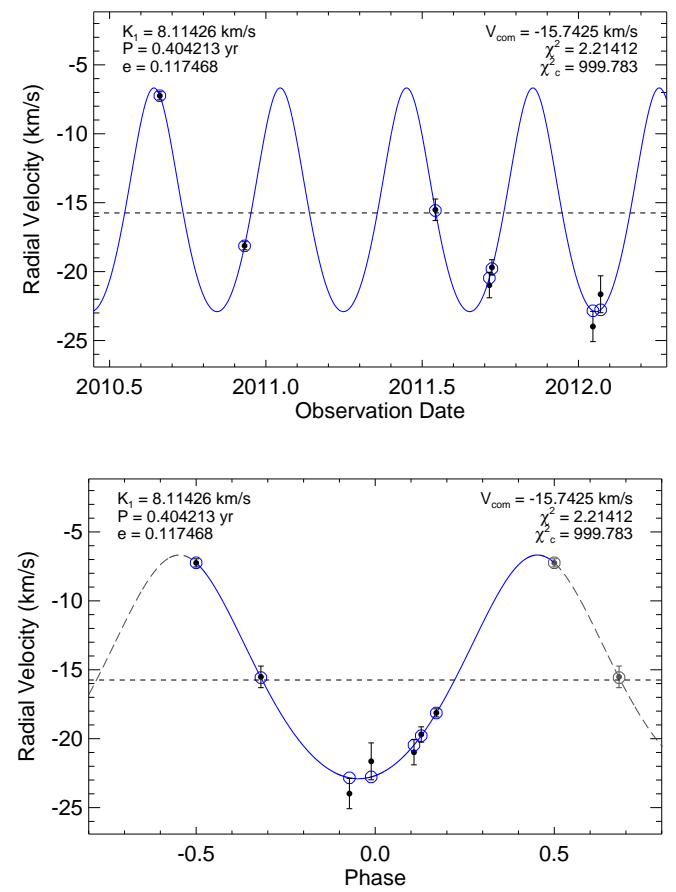

FIG. 10.- Best-fit primary radial velocity orbit solution for SDSS J0006-0852AB based on our Case A MCMC fit. The top panel shows the full radial velocity curve with measurements (solid circles with error bars) as a function of observation date; the bottom panel shows the velocity curve as a function of phase. Open circles indicate the expected radial velocity at a given observational epoch based on the best-fit model (blue lines). Orbital parameters $K_{1}, P, e$, and $V_{C O M}$ (horizontal dashed line) are listed, as is the $\chi^{2}$ value for the fit and for a constant velocity solution $\left(\chi_{c}^{2}\right)$.

tionary model constraints (Case C).

\subsection{Results}

Figure 10 displays the best-fit orbital solution for our Case A MCMC analysis. The observed radial velocities are well-fit to a low-eccentricity $(e=0.12)$, short-period $(0.404 \mathrm{yr}=148$ days $)$ orbit, with the first and last observations taken around maximum elongation. The inferred center-of-mass velocity, $-15.7 \mathrm{~km} \mathrm{~s}^{-1}$, is consistent with the measured velocity of LP 704-48, although this is not surprising for our Case A analysis given the $V_{C O M}$ constraint. With a $\chi^{2}=2.21$, this solution is an excellent fit to the data, and suggests that the uncertainties on the radial velocities may even be slightly overestimated. The best case solutions for our Case B and Case $\mathrm{C}$ analyses have similar parameters, notably yielding the same $V_{C O M}$ values without a constraint, and comparable $\chi^{2}$ values. Figure 11 displays a representative relative astrometric orbit for the best-fit Case A solution. The apparent separation of the two components based on the solution never exceeds 10 mas, making SDSS J0006-0852AB unresolvable with current AO or space-based instrumentation.

Marginalized individual and joint probability distributions for various orbit parameters and parameter pairs were derived by first computing a weight $\wp$ for each solution $i$ in the chain based on its $\chi^{2}$ value relative to the minimum $\chi^{2}$ of the chain (i.e., the best-fit solution):

$$
\wp_{i}=e^{-\left(\chi^{2}{ }_{i}-\min \left[\left\{\chi^{2}\right\}\right]\right) / 2} .
$$




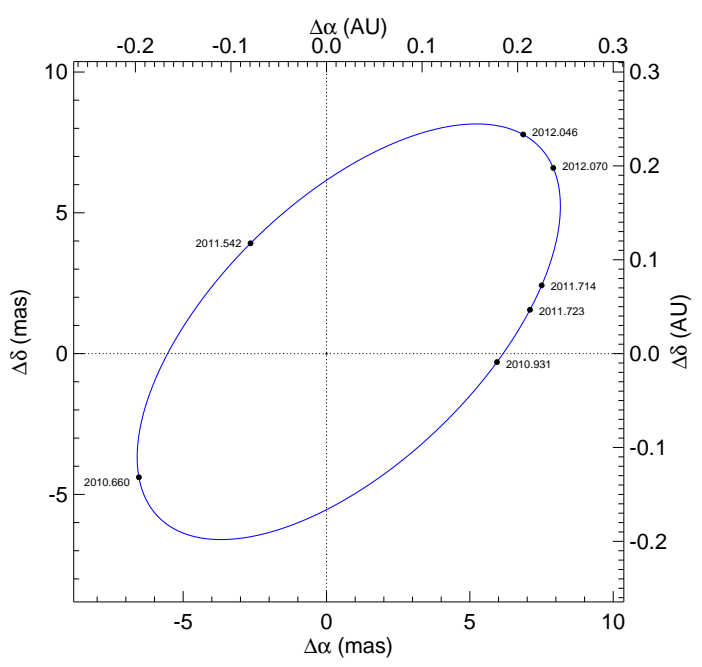

FIG. 11. - Predicted relative astrometric orbit of the LP 70448/SDSS J0006-0852AB system based on the best-fit radial velocity orbit, and assuming $i=i_{\min }$ and an arbitrary longitude of nodes. The primary is located at the origin. Both apparent (bottom and left axes) and projected (top and right axes) coordinates are shown, and epochs at which radial velocity measurements were obtained are labeled.

TABLE 10

Inferred Orbital PARAMETERS For SDSS J0006-0852AB

\begin{tabular}{lccc}
\hline \hline \multicolumn{1}{c}{ Parameter } & Case A & Case B & Case C \\
& & & \\
\hline Minimum $\chi^{2}$ & 2.21 & 2.38 & 1.98 \\
Period (yr) & $0.404 \pm 0.004$ & $0.407 \pm 0.009$ & $0.407 \pm 0.007$ \\
Period (dy) & $147.6 \pm 1.5$ & $148.7 \pm 3.3$ & $148.7 \pm 2.6$ \\
Eccentricity & $0.10 \pm 0.07$ & $0.22 \pm 0.16$ & $0.10 \pm 0.06$ \\
$\omega\left(^{\circ}\right)$ & $-0.3 \pm 2.1$ & $-67 \pm 4$ & $-0.1 \pm 2.2$ \\
$\mathrm{~T}_{0}(\mathrm{MJD})$ & $55421 \pm 3$ & $55421 \pm 5$ & $55421 \pm 4$ \\
$K_{1}\left(\mathrm{~km} \mathrm{~s}^{-1}\right)$ & $8.2 \pm 0.4$ & $8.5 \pm 0.6$ & $8.4 \pm 0.6$ \\
$\left.V_{C O M}(\mathrm{~km} \mathrm{~s})^{-1}\right)$ & $-15.7 \pm 0.2$ & $-16.4 \pm 0.7$ & $-15.9 \pm 0.5$ \\
$f_{M}\left(\mathrm{M}_{\odot}^{1 / 3}\right)$ & $0.202 \pm 0.010$ & $0.205 \pm 0.013$ & $0.202 \pm 0.010$ \\
$a_{1} \sin i(\mathrm{AU})$ & $0.111 \pm 0.005$ & $0.122 \pm 0.011$ & $0.112 \pm 0.018$ \\
$a(\mathrm{AU})^{\mathrm{a}}$ & $0.286 \pm 0.009$ & $0.288 \pm 0.010$ & $\cdots$ \\
Minimum $i\left(^{\circ}\right)^{\mathrm{a}}$ & $61 \pm 5$ & $62 \pm 6$ & $\cdots$ \\
Minimum Age $(\mathrm{Gyr})^{\mathrm{a}}$ & $3.7 \pm 0.8$ & $4.0 \pm 1.0$ & $\cdots$ \\
& & & \\
\hline
\end{tabular}

Note. - Case A incorporates constraints on both $V_{C O M}$ and $f_{M}$; Case B drops the $V_{C O M}$ constraints; Case C drops both $V_{C O M}$ and $f_{M}$ constraints; see Section 5.2 .

a Assuming a combined system mass of $0.144 \pm 0.013 \mathrm{M}_{\odot}$ based on the evolutionary models of Burrows et al. (2001) and Baraffe et al. 2003.

For computational ease, we excluded all parameter sets with $\wp<10^{-4}$, which left of order $10^{5}$ solutions per Case ${ }^{14}$. For individual parameters, we divided the full range of parameter values $\left\{\theta_{j}\right\}$ into 30 bins, and for each bin summed the probabilities of all solutions with $\theta_{j}$ in that bin. The resulting marginalized probability distributions were normalized and then fit with Gaussians to determine parameter means and standard deviations. For parameter pairs, we performed the same analysis, dividing the ranges of both parameters into 30 bins and summing the probabilities of those solutions whose pa-

\footnotetext{
14 This pruning is equivalent to the common practice of excluding some fraction of the initial steps in a given MCMC chain; see for example Ford (2005).
}

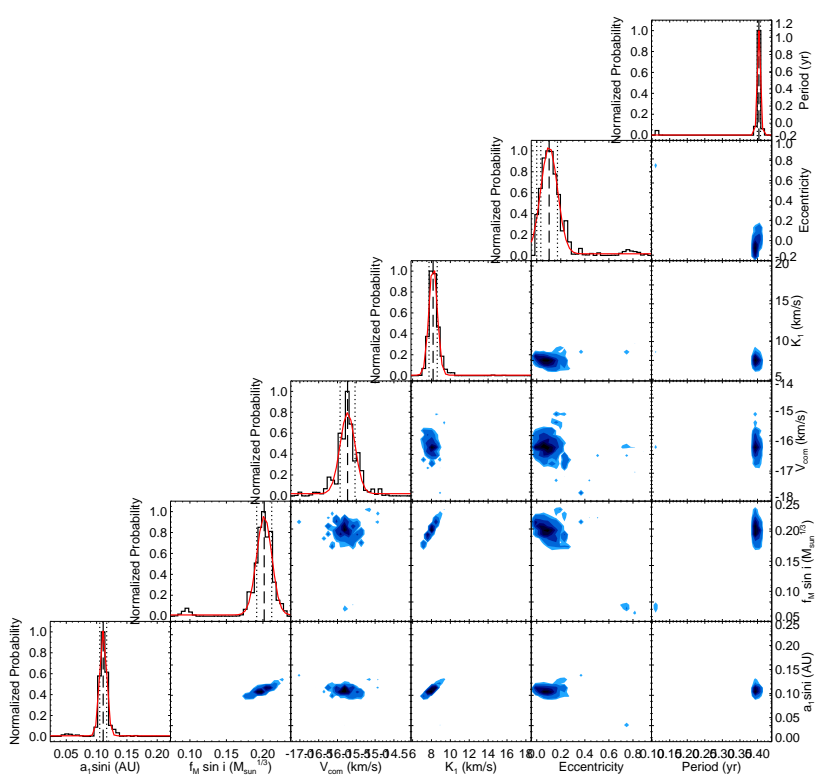

FIG. 12.- Probability distributions of derived orbit parameters for SDSS J0006-0852AB based on our Case A MCMC fit. The leftmost panels show the marginalized probability distributions of (from top to bottom) period (in yr), eccentricity, primary radial velocity semi-amplitude $\left(K_{1}\right.$ in $\left.\mathrm{km} \mathrm{s}^{-1}\right)$, center of mass velocity $\left(V_{C O M}\right.$ in $\left.\mathrm{km} \mathrm{s}^{-1}\right)$, mass function $\left(f_{M}=\mathrm{M}_{2} \sin i /\left(\mathrm{M}_{1}+\mathrm{M}_{2}\right)^{2 / 3}\right.$ in $\mathrm{M}_{\odot}^{1 / 3}$ ), and primary semimajor axis ( $a_{1} \sin i$ in $\left.\mathrm{AU}\right)$. Gaussian fits to these distributions used to determine mean values and standard deviations are shown in red. The other panels display joint probabilities distributions between parameter pairs, which shading from =light to dark blue tracing factors of $0.05,0.1,0.3,0.5,0.7$ and 0.9 relative to the best-fit solution (see Eqn. 8).

rameters simultaneously satisfy both bin ranges.

As summarized in Table [10, we derive fairly stringent $(\lesssim 10 \%)$ constraints on the orbital period $(0.404 \pm 0.004 \mathrm{yr}, 147.6 \pm 1.5$ days $)$, eccentricity $(0.10 \pm 0.07)$, and $K_{1}\left(8.2 \pm 0.4 \mathrm{~km} \mathrm{~s}^{-1}\right)$, and determine $\omega$ to within $2^{\circ}$ and periapse passage $T_{0}$ to within 3 days (periapse occurred 6 days prior to our first observation). These parameters yield $f_{M} \sin i=0.202 \pm 0.010 \mathrm{M}_{\odot}^{1 / 3}$, which is near the maximum $f_{M}$ allowed from the evolutionary models (Eqn 7). Our determination of $V_{C O M}$ $\left(-15.7 \pm 0.2 \mathrm{~km} \mathrm{~s}^{-1}\right)$ is consistent with, and more accurate than, the measured radial velocity of LP 704-48. Inferred parameters for our Case B and Case C analyses were identical to within the uncertainties. We also derived the primary semi-major axis of the system

$$
a_{1} \sin i=\frac{P K_{1}}{2 \pi \sqrt{1-e^{2}}}=0.111 \pm 0.005 \mathrm{AU}
$$

for Case A, with the other Cases giving statistically equivalent results. For comparison, the orbit of 2MASS J0320-0446AB, which is also nearly circular, has a period 1.7 times longer and a primary semi-major axis 1.4 times wider than SDSS J0006-0852AB (Blake et al. 2010). Our marginalized parameter pair distributions show that $P, e$ and $K_{1}$ values are uncorrelated, while $f_{M} \sin i$ and $a_{1} \sin i$ values show an expected correlation with $K_{1}$.

For our analyses that included evolutionary mass constraints, Cases A and B, we determined model-dependent minimum inclinations and system ages (Figure 13). From Eqn 7 we derived $i_{\min }=61^{\circ} \pm 5^{\circ}$ (Case A), with so- 

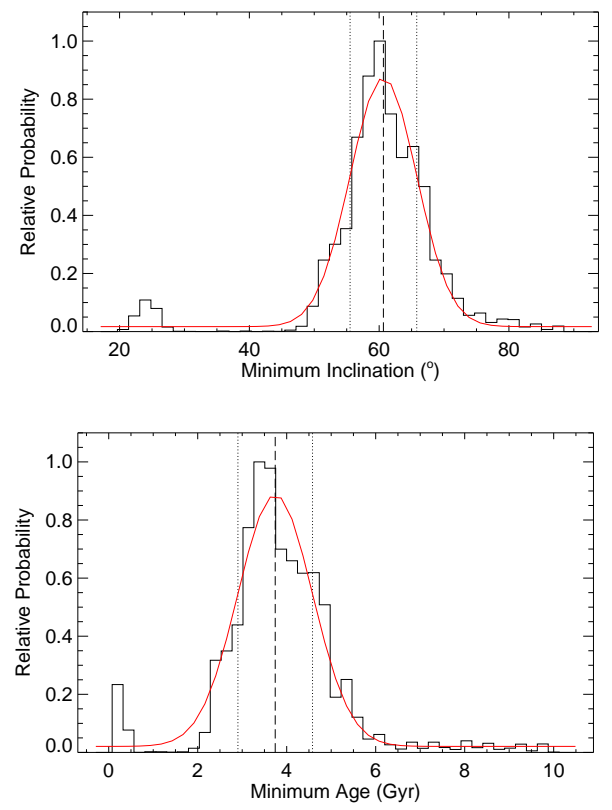

FIG. 13.- Marginalized probability distributions of the minimum orbital inclination (top, in degrees) and minimum system age (bottom, in Gyr) for SDSS J0006-0852AB based on our Case A MCMC fit. These assume component masses inferred from the spectral types and evolutionary models of Baraffe et al. (2003) (see Figure 9).

lutions up to $70^{\circ}$ being significant. However, eclipses remain unlikely for this pair, requiring an inclination $i>89.815$ Constraints on system age is also based on the requirement that $\sin i \leq 1$, which implies a minimum value for the time-dependent $f_{M}$ (Figure 9). Our marginalized probability distribution for the minimum age for Case A peaks at $3.7 \pm 0.8 \mathrm{Gyr}$, consistent with the $8_{-1.0}^{+0.5}$ Gyr minimum age inferred for LP 704-48 based on its lack of $\mathrm{H} \alpha$ emission. Formally adopting a system age of 3-10 Gyr limits the mass of the secondary to 0.049$0.064 \mathrm{M}_{\odot}$, the total system mass to $0.131-0.147 \mathrm{M}_{\odot}$, and the mass ratio of the components to $0.60 \leq q \leq$ 0.78 16. The total system mass can be combined with the period to infer a (model-dependent) semimajor axis of $0.286 \pm 0.009 \mathrm{AU}$. This makes SDSS J0006-0852AB the third tightest VLM binary discovered to date, behind the spectroscopic binary PPL 15AB (Basri \& Martín 1999; $a=0.03 \mathrm{AU}$ ) and the $1 \mathrm{Myr}$ eclipsing brown dwarf binary 2MASS J05352184-0546085 (hereafter 2MASS J0535-0546; Stassun et al. 2006; $a=0.04$ AU), and tighter than 2MASS J0320-0446 $(a=0.4$ AU; Blake et al. (2010)).

\section{DISCUSSION}

\subsection{Very Low Mass Triples}

The LP 704-48/SDSS J0006-0852AB system joins a growing list of VLM triples in which all three components have masses near or below $0.1 \mathrm{M}_{\odot}$ (assuming a

\footnotetext{
15 The geometric probability that one component will eclipse is $\frac{R_{1}+R_{2}}{a} \frac{1+2 e / \pi}{1-e^{2}} \approx 0.4 \%$, assuming $R_{1}=R_{2}=R_{\text {Jupiter }}$.

16 This is based on the evolutionary models of Baraffe et al. (2003). The models of Saumon \& Marlev (2008) yield similar values; the models of Burrows et al. (2001) yield $\mathrm{M}_{2}=0.055$ $0.072 \mathrm{M}_{\odot}, \mathrm{M}_{1}+\mathrm{M}_{2}=0.139-0.157 \mathrm{M}_{\odot}$, and $q=0.66-0.85$.
}

mass of $0.093 \mathrm{M}_{\odot}$ for LP 704-48 based on the evolutionary models used above). Formally defining the category "VLM triple" as a bound system of three hydrogenrich objects with a total mass of $0.3 \mathrm{M}_{\odot}$ or less, we list the seven candidate and confirmed systems that fall into this category in Table 11. With the exception of LHS 1070ABC, these systems are strongly hierarchical, with a ratio of outer to inner separations ranging from $\sim 5$ for LP $714-37 \mathrm{ABC}$ to $\sim 2900$ for LP 70448/SDSS J0006-0852AB. They also have estimated component masses that are comparable to each other, although this may be a selection bias (sensitivity limits) and subject to the typically large uncertainties in mass estimates for substellar dwarfs. Three of these systems have total estimated masses $\mathrm{M}_{t o t} \lesssim 0.15 \mathrm{M}_{\odot}$, although their tertiary components have not been solidly confirmed. DENIS-P J020529.0-115925ABC (hereafter DENIS J0205-1159AB(C); Delfosse et al. 1997; Koerner et al. 1999; Bouv et al. 2005) was resolved into a $\sim 7$ AU binary by AO and HST imaging. Evidence for a third component was reported by Bouy et al. (2005), but only on the basis of persistent residuals in point spread function fitting of the resolved pair in multiepoch HST images. 2MASS J08503593+1057156ABC (hereafter 2MASS J0850+1057AB(C); Kirkpatrick et al. 1999; Reid et al. 2001) was resolved as a 0.16 binary with HST, and subsequently identified as a candidate triple based on the later classification of its primary, despite this component being roughly a magnitude brighter in the near-infrared (Burgasser et al. 2011a). This result has been called into question by Dupuy \& Liu (2012b), however, based on reanalysis of the combined-light spectrum. Kelu $1 \mathrm{AB}(\mathrm{C})$ (Ruiz et al. 1997; Martin et al. 1999; Liu \& Leggett 2005; Gelino et al. 2006) was resolved as a 6 AU visual double with $\mathrm{AO}$ and HST imaging, and its primary identified as an unresolved ( $<4$ AU) L/T spectral binary by Stumpf et al. (2008), although there has been no evidence of RV variability in the combined-light system at the $1-3 \mathrm{~km} \mathrm{~s}^{-1}$ level (Basri \& Reiners 2006; Blake et al. 2010). Given the uncertain nature of these triple candidates, we argue that LP 704-48/SDSS J0006-0852AB is currently the lowestmass triple verified through multiple techniques. It is also the only VLM triple system in Table 11] for which multiple orbits of the tight inner binary have been observed.

The LP 704-48/SDSS J0006-0852AB system shares much in common with two recently uncovered, but slightly more massive, low-mass triples containing transiting substellar components: NLTT 41135AB/NLTT 41136 (Irwin et al. 2010) and LHS 6343ABC (Johnson et al. 2011). Both are similarly composed of relatively wide M-dwarf pairs (55 AU and $20 \mathrm{AU}$, respectively) with one component hosting a tightly-orbiting (0.02 $\mathrm{AU}$ and $0.08 \mathrm{AU})$ substellar mass $\left(0.03 \mathrm{M}_{\odot}\right.$ and $\left.0.06 \mathrm{M}_{\odot}\right)$ companion. For these systems, the component separations are roughly an order of magnitude smaller than for LP 70448/SDSS J0006-0852AB, but the relative inner to outer separations and component masses are comparable. Moreover, based on evolutionary models, the tertiaries of these systems are also likely to be $\mathrm{T}$ dwarfs. The fact that three such systems have been identified in the span of three years suggests that such low-mass 
TABLE 11

Confirmed and Candidate Very Low Mass Triples $\left(\mathrm{M}_{t o t} \lesssim 0.3 \mathrm{M}_{\odot}\right)$

\begin{tabular}{|c|c|c|c|c|c|c|c|c|c|c|c|}
\hline \multicolumn{2}{|c|}{ Components $^{\mathrm{a}}$} & \multicolumn{3}{|c|}{ Spectral Types } & \multicolumn{4}{|c|}{ Estimated Masses $\left(\mathrm{M}_{\odot}\right)$} & \multicolumn{2}{|c|}{ Separations (AU) } & \multirow[t]{2}{*}{ Ref } \\
\hline A & $\mathrm{BC}$ & A & B & $\mathrm{C}$ & A & B & $\mathrm{C}$ & Total & $\mathrm{A}-\mathrm{BC}$ & $\mathrm{BC}$ & \\
\hline LP 714-37A & LP 714-37BC & M5.5 & M8 & M8.5 & 0.11 & 0.09 & 0.08 & 0.28 & $36 \pm 5$ & $6.8 \pm 0.9$ & 1 \\
\hline LHS 1070A & LHS 1070BC & M5.5 & M8.5 & M9 & 0.115 & 0.08 & 0.077 & 0.27 & $\sim 12^{\mathrm{b}}$ & $3.57 \pm 0.07$ & 2,3 \\
\hline LP 213-68 & LP $213-67 \mathrm{AB}$ & M8 & M8 & L0 & 0.09 & 0.09 & 0.084 & 0.27 & $340 \pm 60$ & $2.9 \pm 0.6$ & 4,5 \\
\hline LP 704-48 & SDSS J0006-0852AB & M7 & M8.5 & $\mathrm{T} 5$ & 0.092 & 0.083 & 0.056 & 0.23 & $820 \pm 120$ & $0.286 \pm 0.009$ & 6 \\
\hline DENIS J0205-1159A & DENIS J0205-1159B(C) ${ }^{\mathrm{c}}$ & L5 & L8 & T0: & 0.06 & 0.05 & 0.04 & 0.15 & $7 \pm 1$ & $\sim 1.3$ & 7 \\
\hline 2MASS J0850+1057B & $2 \mathrm{MASS} \mathrm{J} 0850+1057 \mathrm{~A}(\mathrm{C})^{\mathrm{c}}$ & L6 & L7 & L7: & 0.05 & 0.05 & 0.05 & 0.15 & $6.0 \pm 0.9$ & $<4$ & 8 \\
\hline Kelu 1B & Kelu $1 \mathrm{~A}(\mathrm{C})^{\mathrm{c}}$ & L3p & $\mathrm{L} 0.5$ & T7: & 0.05 & 0.06 & 0.02 & 0.13 & $6.4_{-1.3}^{+2.4}$ & $<4$ & 9 \\
\hline
\end{tabular}

References. - (1) Phan-Bao et al. (2006); (2) Leinert et al. (2001); (3) Seifahrt et al. (2008); (4) Gizis et al. (2000a); (5) Close et al. (2003); (6) This paper; (7) Bouv et al. (2005); (8) Burgasser et al. (2011a); (9) Stumpf et al. (2008).

a For this table, we refer to the outermost component as "A" and the inner binary as "BC" irrespective of mass or designation.

b Scaling from the inner semi-major axis using the period-mass ratio: $\left(a_{\text {out }} / a_{\text {in }}\right)^{3}=\left(P_{\text {out }} / P_{\text {in }}\right)^{2} \times\left(M_{\text {out }} / M_{\text {in }}\right)$, and values from Seifahrt et al. (2008).

c Candidate triples with unconfirmed third component.

triple configurations may be quite common, particularly among wide VLM pairs (e.g., Law et al. 2010), although a robust survey is needed to quantify the incidence of such systems.

\subsection{Stability of the LP 704-48/SDSS J0006-0852AB System}

The LP 704-48/SDSS J0006-0852AB system is at an extremum among the VLM triples listed in Table 11 in that it has both the widest outer separation and the smallest (measured) inner separation in the sample. The outer pairing is remarkable for a system with $\mathrm{M}_{t o t} \lesssim 0.25 \mathrm{M}_{\odot} ;$ currently, only three other VLM field binaries are known to have projected separations $>500$ AU17 The gravitational binding energy of LP 704-48 and SDSS J0006-0852AB, $\left|E_{b}\right| \lesssim$ $(2-3) \times 10^{41} \mathrm{erg}$, is low but not unprecedented. Recently, several wide multiples of comparable total mass and binding energy have been found (e.g., Dhital et al. 2010; Law et al. 2010), including high-order systems such as NLTT $20346 \mathrm{AB} / 2 \mathrm{MASS}$ J0850359+105716AB(C) $\left(\left|E_{b}\right| \approx 0.4 \times 10^{41}\right.$ erg; Kirkpatrick et al. 1999; Reid et al. 2001; Burgasser et al. 2011a; Faherty et al. 2011) and G 124-62/DENIS-P J144137.3-094559AB $\left(\left|E_{b}\right| \approx 3 \times\right.$ $10^{41}$ erg; Martín et al. 1999, 2006; Bouy et al. 2003; Seifahrt et al. 2005), both of which contain substellar components. In part, it is the additional mass of the $\mathrm{T}$ dwarf tertiary that pushes the LP 70448/SDSS J0006-0852AB system into a "normal" regime in mass/separation space (see Faherty et al. 2011). This component also contributes to the long-term stability of the wide pair to external perturbation (Weinberg et al. 1987; Dhital et al. 2010).

Because of the large difference in outer to inner separations, LP 704-48/SDSS J0006-0852AB is also exceptionally stable to internal dynamical disruption. Internal stability can be quantified with the criteria of Eggleton \& Kiseleva (1995), who examined the ratio of outer periapse $a_{\text {out }}\left(1-e_{\text {out }}\right)$ to inner apoapse $a_{\text {in }}\left(1+e_{\text {in }}\right)$ for triple star systems. For simplicity, we drop the ec-

17 Koenigstuhl-1 at 1800 AU (Caballero 2007), 2MASS J0126555-502239 at 5100 AU; (Artigau et al.|2007, 2009) and 2MASS J12583501+4013083 at 6700 AU (Radigan et al. 2009).

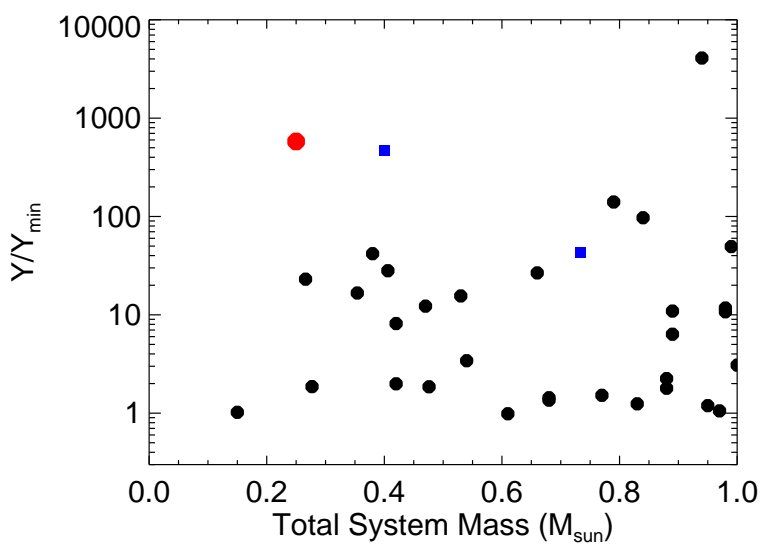

FIG. 14. - Stability ratio $Y / Y_{\min }$ as a function of total system mass for a sample of low mass triple systems drawn from the Multiple Star Catalog (Tokovinin 1997); Faherty et al. (2011); and this paper. The LP 704-48/SDSS J0006-0852AB system is indicated by the large red circle at left; the eclipsing triples NLTT $41135 \mathrm{AB}+$ NLTT 41136 (Irwin et al. 2010) and LHS 6343ABC (Johnson et al. 2011) are indicated by blue squares.

centricity terms and approximate the ratio $Y=a_{\text {out }} / a_{\text {in }}$, using as $a_{\text {out }}$ the projected separation of the wide pairing. For LP 704-48/SDSS J0006-0852AB, $Y \approx 2900$, a value several orders of magnitude greater the critical ratio

$$
Y_{\min }=1+\frac{3.7}{q_{\text {out }}^{1 / 3}}+\frac{2.2}{1+q_{\text {out }}^{1 / 3}}+1.4 q_{\text {in }}^{1 / 3} \frac{q_{\text {out }}^{1 / 3}-1}{1+q_{\text {out }}^{1 / 3}},
$$

which is $\approx 5$ for this system, assuming $q_{\text {in }} \equiv \mathrm{M}_{B} / \mathrm{M}_{A} \approx$ 0.7 for SDSS J0006-0852AB and $q_{\text {out }} \equiv\left(\mathrm{M}_{A}+\right.$ $\left.\mathrm{M}_{B}\right) / \mathrm{M}_{L P}{ }_{704-48} \approx 1.618$ Figure 14 compares the ratio $Y / Y_{\min }$ for LP 704-48/SDSS J0006-0852AB and other low-mass triples listed in the most recent version (April 2010) of the Multiple Star Catalog (Tokovinin 1997), Faherty et al. (2011) and Table 11. By this metric, LP 704-48/SDSS J0006-0852AB stands out as the most internally stable low-mass triple known, slightly

\footnotetext{
18 These estimates also imply a period ratio $X=$ $Y^{1.5}\left(\frac{q_{\text {out }}}{q_{\text {out }}+1}\right)^{0.5} \approx 10^{5}$.
} 
more stable than the NLTT 41135 AB/NLTT 41136 system (Irwin et al. 2010). With such a large semi-major axis ratio, we note that $\mathrm{N}$-body simulations find the outer orbits of such systems are likely to have a significant eccentricity (Sterzik \& Tokovinin 2002), so the ratio of outer periapse to inner apoapse may be smaller than the semimajor axis ratio used here.

\subsection{On the Formation of LP 704-48/SDSS J0006-0852AB}

Given the now established existence of a handful of VLM triples like LP 704-48/SDSS J0006-0852AB, it is worth examining whether such systems and their characteristics match the predictions of star and brown dwarf formation models. We emphasize that current samples are far from statistically robust or complete, and detection biases may be significant.

Despite the low production rate of VLM multiples in general, current models do produce VLM higher-order systems. A commonly-ascribed mechanism is dynamical scattering in the post-accretion phase of a young cluster (Sterzik \& Durisen 2003; Delgado-Donate et al. 2004; Goodwin et al. 2004). In their study of small N-body cluster dynamics, Sterzik \& Durisen (2003) found that $11 \%$ of their simulated systems formed triple and higherorder multiples, and over $40 \%$ of the triples contained at least one brown dwarf component. Most of these systems were widely separated and hierarchical, typically with the distant companion being of low mass (in systems containing two brown dwarfs, the distant companion was a brown dwarf in $90 \%$ of cases). However, all wide triples $(a \gtrsim 100 \mathrm{AU})$ with brown dwarf components had extremely low mass ratios $(q<0.3)$, whereas the systems listed in Table 11 are largely composed of near-equal mass components. Moreover, this simulation proved unable to generate VLM binaries tighter than $\sim 1 \mathrm{AU}$, and the authors conclude that dynamical interactions alone cannot explain the existence of such systems (which at the time included only PPl 15). Hence, it would appear that dynamics alone is incapable of producing VLM multiples like LP 704-48/SDSS J0006-0852AB, a conclusion that has been reached for more massive higher-order multiples as well (Tokovinin 2008).

Accretion plays an important role in the formation of multiple systems, as a mechanism for angular momentum exchange and dissipation, and driving binaries to near-equal masses (e.g., Bate et al. 2002). Bate (2012) recently examined VLM multiplicity in radiative hydrodynamic simulations of molecular cloud collapse, which incorporates both gas accretion (with some radiative feedback) and dynamics, albeit over shorter periods than pure N-body simulations due to computational constraints. The Bate study produced three low-mass triple systems $\left(\mathrm{M}_{t o t} \lesssim 0.6 \mathrm{AU}\right)$, all of which were hierarchical with either the high mass or middle mass component at wide separation. While an exact clone to LP 70448/SDSS J0006-0852AB was not generated, one system composed of a $0.15 \mathrm{M}_{\odot}$ plus $0.03 \mathrm{M}_{\odot}, 14 \mathrm{AU}$ inner binary with a $0.07 \mathrm{M}_{\odot}$ wide companion at $194 \mathrm{AU}$ is a reasonable analog, although the large inner eccentricity of this triple $(e=0.8)$ may ultimately lead to its dissolution. One fundamental shortcoming of this study is the $\sim 1 \mathrm{AU}$ separation range limit on close interactions, again due to computational constraints. Bate (2009) examined VLM binary statistics at ten times higher resolution but over a shorter timeframe and employing slightly different gas physics. While they could successfully form 0.1-1.0 AU VLM binaries in these simulations, no analysis was made of higher-order multiples.

The fragmentation of circumstellar disks around massive stars is another proposed mechanism for forming VLM stars and brown dwarfs (Stamatellos \& Whitworth 2008, 2009). Unfortunately, the predicted binary rate for VLM systems created in these environments is very low ( $\sim 8 \%$ for ejected systems) and no low-mass triples have been created in simulations to date. Furthermore, while the separation distribution of simulated substellar pairs peaks in a range that is close to that inferred for SDSS J0006-0852AB $(0.3<a<0.6$ AU $)$, these binaries tend to have very large eccentricities $(e>0.7)$, a feature not seen in any of the VLM spectroscopic binaries identified to date. As such, disk fragmentation does not appear to be a viable mechanism for making triples like LP 704-48/SDSS J0006-0852AB.

It is important to emphasize that current models fall short in reproducing very short-period, loweccentricity VLM pairs like SDSS J0006-0852AB and 2MASS J0320-0446AB. Such systems are necessarily the product of dynamical and dissipative evolution, as opacity-limited fragmentation constrains the initial separations of self-gravitating masses to $\gtrsim 10 \mathrm{AU}$ (Larson 1969; Low \& Lynden-Bell 1976). Bate et al. (2002) resolves this problem through a combination of dynamical interactions and accretion from a circumbinary/circumtertiary disk, although again these simulations fail to produce stable VLM multiples. Indeed, three-body encounters more often than not disrupt VLM multiples (Moeckel \& Bate 2010). A more intriguing mechanism for SDSS J0006-0852AB is KozaiLidov eccentricity perturbations induced by LP 70448, followed by circularization through tidal friction (KCTF; Kozai 1962; Harrington 1968; Kiseleva et al. 1998; Fabrvcky \& Tremaine 2007; Socrates et al. 2012). This mechanism has been proposed to explain the high fraction of spectroscopic binaries with tertiary companions $(96 \%$ for SBs with $P<3$ dy; Tokovinin et al. 2006; see also Raghavan et al. 2010). KCTF predicts end states with roughly circular inner orbits and large period ratios $\left(X \gtrsim 10^{3}-10^{4}\right.$; Fabrvcky \& Tremaine 2007), similar to SDSS J0006-0852AB. Unfortunately, the timescale for eccentricity pumping is long for LP 70448/SDSS J0006-0852AB $\left(\tau \sim P_{\text {out }}^{2} / P_{\text {in }} \approx 3 \mathrm{Gyr}\right)$, while tidal circularization is extremely inefficient at the current orbital separation of SDSS J0006-0852AB 19 (Hut 1981; Meibom \& Mathieu 2005). Thus, it appears that $\mathrm{KCTF}$ is probably not responsible for the current config-

19 Using Eqns 50 and 52 from Hut (1981), the timescale for circularization:

$$
t_{\text {circ }}=\frac{0.0024}{k q}\left(\frac{a_{0}}{R_{1}}\right)^{8} \frac{P_{0}}{\tau} P_{0}
$$

where $k$ is the primary's Love number, $q$ the mass ratio, $a_{0}$ and $P_{0}$ the final semimajor axis and period, $R_{1}$ the primary's radius and $\tau$ the tidal lag with respect to the displacement between primary and secondary. Assuming $k \sim 0.5$ (i.e., comparable to Jupiter and Saturn), $q=0.7, a_{0} / R_{1}=0.29 \mathrm{AU} / R_{\text {Jupiter }} \approx 620$ and $P_{0}=0.4 \mathrm{yr}$ and $\tau \approx 0.1 P_{0}$, we derive $t_{\text {circ }} \approx 6 \times 10^{20} \mathrm{yr}$, far too long to have played a role in the dynamic evolution of SDSS J0006-0852AB. 
uration of this system, nor presumably for the isolated tight VLM binaries PPl 15AB, 2MASS J0535-0546AB or 2MASS J0320-0446AB.

A final possibility is that LP 704-48 has played no role in the evolution of the SDSS J0006-0852AB binary, which could have shrunk and circularized through dissipative interactions with an early circumbinary disk or a closer encounter resulting in an ejection. The wider pairing with LP 704-48 may simply be a normal (albeit rare) outcome of cloud fragmentation or parallel trajectories during the dissolution of the natal cluster (Kouwenhoven et al. 2010; Moeckel \& Bate 2010). LP 704-48 and SDSS J0006-0852AB may be more akin to stellar cousins that stellar siblings.

\section{SUMMARY}

We have identified a hierarchical triple system, LP 70448/SDSS J0006-0852AB, composed of a tight $\left(a_{i n}=\right.$ 0.28 AU) M9 plus T5 \pm 1 binary straddling the hydrogen burning mass limit, with a wide $\left(a_{\text {out }} \approx 820 \mathrm{AU}\right)$, inactive M7 co-moving companion. The inner pair is found to be both a spectral (blended light) binary and a radial velocity variable. By combining model-dependent mass constraints from the component spectral types, the radial velocity of LP 704-48, and orbit model fits of the RV data (spanning more than three orbital periods), we have determined the orbital parameters of SDSS J0006-0852AB, including constraints on the orbit inclination and the system's age ( $\gtrsim 3$ Gyr). The latter is consistent with the age inferred for LP 70448 based on its lack of $\mathrm{H} \alpha$ emission ( $8_{-1.0}^{+0.5} \mathrm{Gyr}$ ). SDSS J0006-0852AB is currently the third tightest VLM binary known, and its identification verifies that the spectral binary method can probe a relatively unexplored separation range among VLM multiples. With LP 70448 , this system forms the lowest-mass triple identified to date whose tertiary is unambiguously detected, and is one of seven confirmed and candidate VLM triples whose total mass is $\lesssim 0.3 \mathrm{M}_{\odot}$. While current star formation models are capable of producing triples with substellar components, we find that they do not yet produce systems that replicate VLM triples like LP 70448/SDSS J0006-0852AB. In particular, dynamical interactions alone do not appear to be responsible for the close separation of the SDSS J0006-0852AB pair, while disk fragmentation and hydrodynamic models have yet to produce comparable systems. We also rule out Kozai-Lidov perturbations and tidal circularization for the current configuration of this system. As most of the $P<1$ yr VLM binaries found to date are isolated pairs, we speculate that LP 704-48 and SDSS J0006-0852AB formed as two separate systems bound only by proximity and/or common motion after cluster dissolution. Assessing whether this is the primary mode for VLM triple formation, and whether VLM triples are common or rare, will require a more statistically robust survey. Nevertheless, these systems are benchmarks for studying multiple star formation theory; brown dwarf evolutionary models; and age, activity and metallicity diagnostics for VLM stars.

The authors thank Joel Aycock, Scott Dahm, Heather Hershley, Carolyn Parker, Jim Lyke, Julie Rivera, and Greg Wirth at Keck Observatory; Bobby Bus, Bill Golisch and John Rayner at IRTF; and Dave Summers at KPNO for their assistance with the observations. The authors acknowledge helpful comments from Trent Dupuy and Tristan Guillot. AJB acknowledges Rob and Terry Ryan for providing accommodation while completing this manuscript. We also thank our referee, John Johnson, for his prompt and insightful review.

This publication makes use of data products from the Two Micron All Sky Survey, which is a joint project of the University of Massachusetts and the Infrared Processing and Analysis Center/California Institute of Technology, funded by the National Aeronautics and Space Administration and the National Science Foundation. This article has also made use of data from the Sloan Digital Sky Survey. Funding for the SDSS and SDSS-II has been provided by the Alfred P. Sloan Foundation, the Participating Institutions, the National Science Foundation, the U.S. Department of Energy, the National Aeronautics and Space Administration, the Japanese Monbukagakusho, the Max Planck Society, and the Higher Education Funding Council for England. The SDSS Web Site is http://www.sdss.org/. The SDSS is managed by the Astrophysical Research Consortium for the Participating Institutions. The Participating Institutions are the American Museum of Natural History, Astrophysical Institute Potsdam, University of Basel, University of Cambridge, Case Western Reserve University, University of Chicago, Drexel University, Fermilab, the Institute for Advanced Study, the Japan Participation Group, Johns Hopkins University, the Joint Institute for Nuclear Astrophysics, the Kavli Institute for Particle Astrophysics and Cosmology, the Korean Scientist Group, the Chinese Academy of Sciences (LAMOST), Los Alamos National Laboratory, the Max-Planck-Institute for Astronomy (MPIA), the Max-Planck-Institute for Astrophysics (MPA), New Mexico State University, Ohio State University, University of Pittsburgh, University of Portsmouth, Princeton University, the United States Naval Observatory, and the University of Washington. This research has benefitted from the $\mathrm{M}, \mathrm{L}$, and $\mathrm{T}$ dwarf compendium housed at http://DwarfArchives.org and maintained by Chris Gelino, Davy Kirkpatrick, and Adam Burgasser; the the Very-Low-Mass Binaries Archive housed at http://www.vlmbinaries.org and maintained by Nick Siegler, Chris Gelino, and Adam Burgasser; and the SpeX Prism Spectral Libraries, maintained by Adam Burgasser at http://www.browndwarfs.org/spexprism. This research has also made use of the SIMBAD database and VizieR service, operated at CDS, Strasbourg, France.

The authors recognize and acknowledge the very significant cultural role and reverence that the summit of Mauna Kea has always had within the indigenous Hawaiian community. We are most fortunate to have the opportunity to conduct observations from this mountain.

\section{REFERENCES}


Allen, P. R. 2007, ApJ, 668, 492

Artigau, É., Lafrenière, D., Albert, L., \& Doyon, R. 2009, ApJ, 692,149

Artigau, É., Lafrenière, D., Doyon, R., Albert, L., Nadeau, D., \& Robert, J. 2007, ApJ, 659, L49

Baraffe, I., Chabrier, G., Barman, T. S., Allard, F., \& Hauschildt, P. H. 2003, A\&A, 402, 701

Barth, A. J. 2001, in Astronomical Society of the Pacific Conference Series, Vol. 238, Astronomical Data Analysis Software and Systems X, ed. F. R. Harnden Jr., F. A. Primini, \& H. E. Payne, 385

Basri, G., \& Martín, E. L. 1999, AJ, 118, 2460

Basri, G., \& Reiners, A. 2006, AJ, 132, 663

Bate, M. R. 2009, MNRAS, 392, 590

-. 2012, MNRAS, 419, 3115

Bate, M. R., Bonnell, I. A., \& Bromm, V. 2002, MNRAS, 336, 705

Blake, C. H., Charbonneau, D., \& White, R. J. 2010, ApJ, 723, 684

Blake, C. H., Charbonneau, D., White, R. J., Torres, G., Marley, M. S., \& Saumon, D. 2008, ApJ, 678, L125

Bochanski, J. J., Hawley, S. L., Covey, K. R., West, A. A., Reid, I. N., Golimowski, D. A., \& Ivezić, Ż. 2010, AJ, 139, 2679

Bochanski, J. J., Munn, J. A., Hawley, S. L., West, A. A., Covey, K. R., \& Schneider, D. P. 2007a, AJ, 134, 2418

Bochanski, J. J., West, A. A., Hawley, S. L., \& Covey, K. R. 2007b, AJ, 133, 531

Boss, A. P. 2001, ApJ, 551, L167

Bouy, H., Brandner, W., Martín, E. L., Delfosse, X., Allard, F., \& Basri, G. 2003, AJ, 126, 1526

Bouy, H., Martín, E. L., Brandner, W., \& Bouvier, J. 2005, AJ, 129,511

Burgasser, A. J. 2007a, ApJ, 659, 655

—. 2007b, AJ, 134, 1330

-. 2007c, ApJ, 658, 617

Burgasser, A. J., Bardalez-Gagliuffi, D. C., \& Gizis, J. E. 2011a, AJ, 141, 70

Burgasser, A. J., \& Blake, C. H. 2009, AJ, 137, 4621

Burgasser, A. J., Burrows, A., \& Kirkpatrick, J. D. 2006a, ApJ, 639, 1095

Burgasser, A. J., Cruz, K. L., Cushing, M., Gelino, C. R., Looper, D. L., Faherty, J. K., Kirkpatrick, J. D., \& Reid, I. N. 2010, ApJ, 710, 1142

Burgasser, A. J., Geballe, T. R., Leggett, S. K., Kirkpatrick, J. D., \& Golimowski, D. A. 2006b, ApJ, 637, 1067

Burgasser, A. J., Kirkpatrick, J. D., Cruz, K. L., Reid, I. N., Leggett, S. K., Liebert, J., Burrows, A., \& Brown, M. E. 2006c, ApJS, 166, 585

Burgasser, A. J., Kirkpatrick, J. D., Reid, I. N., Brown, M. E., Miskey, C. L., \& Gizis, J. E. 2003, ApJ, 586, 512

Burgasser, A. J., Liu, M. C., Ireland, M. J., Cruz, K. L., \& Dupuy, T. J. 2008a, ApJ, 681, 579

Burgasser, A. J., Looper, D. L., Kirkpatrick, J. D., Cruz, K. L., \& Swift, B. J. 2008b, ApJ, 674, 451

Burgasser, A. J., Looper, D. L., Kirkpatrick, J. D., \& Liu, M. C. 2007a, ApJ, 658, 557

Burgasser, A. J., \& McElwain, M. W. 2006, AJ, 131, 1007

Burgasser, A. J., McElwain, M. W., Kirkpatrick, J. D., Cruz, K. L., Tinney, C. G., \& Reid, I. N. 2004, AJ, 127, 2856

Burgasser, A. J., Reid, I. N., Siegler, N., Close, L., Allen, P., Lowrance, P., \& Gizis, J. 2007b, Protostars and Planets V, 427

Burgasser, A. J., Sitarski, B. N., Gelino, C. R., Logsdon, S. E., \& Perrin, M. D. 2011b, ApJ, 739, 49

Burrows, A., Hubbard, W. B., Lunine, J. I., \& Liebert, J. 2001, Reviews of Modern Physics, 73, 719

Caballero, J. A. 2007, A\&A, 462, L61

Chiu, K., Fan, X., Leggett, S. K., Golimowski, D. A., Zheng, W. Geballe, T. R., Schneider, D. P., \& Brinkmann, J. 2006, AJ, 131,2722

Clark, B. M., Blake, C. H., \& Knapp, G. R. 2012, ApJ, 744, 119

Close, L. M., Siegler, N., Freed, M., \& Biller, B. 2003, ApJ, 587, 407

Cruz, K. L., Burgasser, A. J., Reid, I. N., \& Liebert, J. 2004, ApJ, 604, L61

Cruz, K. L., \& Reid, I. N. 2002, AJ, 123, 2828

Cruz, K. L., Reid, I. N., Liebert, J., Kirkpatrick, J. D., \& Lowrance, P. J. 2003, AJ, 126, 2421
Cushing, M. C., Vacca, W. D., \& Rayner, J. T. 2004, PASP, 116, 362

Delfosse, X., Beuzit, J.-L., Marchal, L., Bonfils, X., Perrier, C., Ségransan, D., Udry, S., Mayor, M., \& Forveille, T. 2004, in Astronomical Society of the Pacific Conference Series, Vol. 318, Spectroscopically and Spatially Resolving the Components of the Close Binary Stars, ed. R. W. Hilditch, H. Hensberge, \& K. Pavlovski, 166-174

Delfosse, X., et al. 1997, A\&A, 327, L25

Delgado-Donate, E. J., Clarke, C. J., \& Bate, M. R. 2003 MNRAS, 342, 926

Delgado-Donate, E. J., Clarke, C. J., Bate, M. R., \& Hodgkin, S. T. 2004, MNRAS, 351, 617

Dhital, S., West, A. A., Stassun, K. G., \& Bochanski, J. J. 2010 AJ, 139, 2566

Dupuy, T. J., \& Liu, M. C. 2012a, ApJS, 201, 19

—. 2012b, ArXiv e-prints

Eggleton, P., \& Kiseleva, L. 1995, ApJ, 455, 640

Fabrycky, D., \& Tremaine, S. 2007, ApJ, 669, 1298

Faherty, J. K., Burgasser, A. J., Bochanski, J. J., Looper, D. L., West, A. A., \& van der Bliek, N. S. 2011, AJ, 141, 71

Fischer, D. A., \& Marcy, G. W. 1992, ApJ, 396, 178

Ford, E. B. 2005, AJ, 129, 1706

Geißler, K., Metchev, S., Kirkpatrick, J. D., Berriman, G. B., \& Looper, D. 2011, ApJ, 732, 56

Gelino, C. R., \& Burgasser, A. J. 2010, AJ, 140, 110

Gelino, C. R., Kulkarni, S. R., \& Stephens, D. C. 2006, PASP, 118,611

Gelman, A., \& Rubin, D. B. 1992, Stat. Sci., 7, 457

Gizis, J. E., Monet, D. G., Reid, I. N., Kirkpatrick, J. D., \& Burgasser, A. J. 2000a, MNRAS, 311, 385

Gizis, J. E., Monet, D. G., Reid, I. N., Kirkpatrick, J. D., Liebert, J., \& Williams, R. J. 2000b, AJ, 120, 1085

Goodwin, S. P., Whitworth, A. P., \& Ward-Thompson, D. 2004, A\&A, 414, 633

Hambly, N. C., Davenhall, A. C., Irwin, M. J., \& MacGillivray, H. T. 2001a, MNRAS, 326, 1315

Hambly, N. C., Irwin, M. J., \& MacGillivray, H. T. 2001b, MNRAS, 326, 1295

Hambly, N. C., MacGillivray, H. T., Read, M. A., Tritton, S. B., Thomson, E. B., Kelly, B. D., Morgan, D. H., Smith, R. E., Driver, S. P., Williamson, J., Parker, Q. A., Hawkins, M. R. S., Williams, P. M., \& Lawrence, A. 2001c, MNRAS, 326, 1279

Harrington, R. S. 1968, AJ, 73, 190

Hawkins, M. R. S., \& Bessell, M. S. 1988, MNRAS, 234, 177

Hawley, S. L., Gizis, J. E., \& Reid, I. N. 1996, AJ, 112, 2799

Hawley, S. L., et al. 2002, AJ, 123, 3409

Hut, P. 1981, A\&A, 99, 126

Irwin, J., Buchhave, L., Berta, Z. K., Charbonneau, D., Latham, D. W., Burke, C. J., Esquerdo, G. A., Everett, M. E., Holman, M. J., Nutzman, P., Berlind, P., Calkins, M. L., Falco, E. E., Winn, J. N., Johnson, J. A., \& Gazak, J. Z. 2010, ApJ, 718, 1353

Joergens, V. 2008, A\&A, 492, 545

Johnson, J. A., Apps, K., Gazak, J. Z., Crepp, J. R., Crossfield, I. J., Howard, A. W., Marcy, G. W., Morton, T. D., Chubak, C., \& Isaacson, H. 2011, ApJ, 730, 79

Jurić, M., Ivezić, Ž., Brooks, A., Lupton, R. H., Schlegel, D., Finkbeiner, D., Padmanabhan, N., Bond, N., Sesar, B., Rockosi, C. M., Knapp, G. R., Gunn, J. E., Sumi, T., Schneider, D. P., Barentine, J. C., Brewington, H. J., Brinkmann, J., Fukugita, M., Harvanek, M., Kleinman, S. J., Krzesinski, J., Long, D., Neilsen, Jr., E. H., Nitta, A., Snedden, S. A., \& York, D. G. 2008, ApJ, 673, 864

Kendall, T. R., Delfosse, X., Martín, E. L., \& Forveille, T. 2004, A\&A, 416, L17

Kirkpatrick, J. D., Looper, D. L., Burgasser, A. J., Schurr, S. D., Cutri, R. M., Cushing, M. C., Cruz, K. L., Sweet, A. C., Knapp, G. R., Barman, T. S., Bochanski, J. J., Roellig, T. L., McLean, I. S., McGovern, M. R., \& Rice, E. L. 2010, ApJS, 190,100

Kirkpatrick, J. D., Reid, I. N., Liebert, J., Cutri, R. M., Nelson, B., Beichman, C. A., Dahn, C. C., Monet, D. G., Gizis, J. E., \& Skrutskie, M. F. 1999, ApJ, 519, 802

Kirkpatrick, J. D., Reid, I. N., Liebert, J., Gizis, J. E., Burgasser, A. J., Monet, D. G., Dahn, C. C., Nelson, B., \& Williams, R. J. 2000, AJ, 120, 447 
Kiseleva, L. G., Eggleton, P. P., \& Mikkola, S. 1998, MNRAS, 300, 292

Kobulnicky, H. A., \& Fryer, C. L. 2007, ApJ, 670, 747

Koerner, D. W., Kirkpatrick, J. D., McElwain, M. W., \& Bonaventura, N. R. 1999, ApJ, 526, L25

Kouwenhoven, M. B. N., Brown, A. G. A., Zinnecker, H., Kaper, L., \& Portegies Zwart, S. F. 2005, A\&A, 430, 137

Kouwenhoven, M. B. N., Goodwin, S. P., Parker, R. J., Davies, M. B., Malmberg, D., \& Kroupa, P. 2010, MNRAS, 404, 1835 Kozai, Y. 1962, AJ, 67, 591

Kraus, A. L., Ireland, M. J., Martinache, F., \& Hillenbrand, L. A. 2011, ApJ, 731, 8

Kurosawa, R., Harries, T. J., \& Littlefair, S. P. 2006, MNRAS, 372,1879

Larson, R. B. 1969, MNRAS, 145, 271

Law, N. M., Dhital, S., Kraus, A., Stassun, K. G., \& West, A. A. 2010, ApJ, 720, 1727

Leggett, S. K. 1992, ApJS, 82, 351

Leinert, C., Jahreiß, H., Woitas, J., Zucker, S., Mazeh, T., Eckart, A., \& Köhler, R. 2001, A\&A, 367, 183

Lépine, S., Rich, R. M., \& Shara, M. M. 2003, AJ, 125, 1598

—. 2007, ApJ, 669, 1235

Liebert, J., \& Burgasser, A. J. 2007, ApJ, 655, 522

Liu, M. C., \& Leggett, S. K. 2005, ApJ, 634, 616

Lodieu, N., Scholz, R.-D., McCaughrean, M. J., Ibata, R., Irwin, M., \& Zinnecker, H. 2005, A\&A, 440, 1061

Looper, D. L., Gelino, C. R., Burgasser, A. J., \& Kirkpatrick, J. D. 2008a, ApJ, 685, 1183

Looper, D. L., Kirkpatrick, J. D., \& Burgasser, A. J. 2007, AJ, 134,1162

Looper, D. L., Kirkpatrick, J. D., Cutri, R. M., Barman, T., Burgasser, A. J., Cushing, M. C., Roellig, T., McGovern, M. R., McLean, I. S., Rice, E., Swift, B. J., \& Schurr, S. D. 2008b, ApJ, 686, 528

Low, C., \& Lynden-Bell, D. 1976, MNRAS, 176, 367

Luhman, K. L., et al. 2007, ApJ, 654, 570

Luyten, W. J. 1980, NLTT Catalogue. Volume_III. 0_to -30_. (University of Minnesota)

Martin, E. L., Brandner, W., \& Basri, G. 1999, Science, 283, 1718

Martín, E. L., Brandner, W., Bouy, H., Basri, G., Davis, J., Deshpande, R., \& Montgomery, M. M. 2006, A\&A, 456, 253

Martín, E. L., Delfosse, X., Basri, G., Goldman, B., Forveille, T., \& Zapatero Osorio, M. R. 1999, AJ, 118, 2466

Maxted, P. F. L., \& Jeffries, R. D. 2005, MNRAS, 362, L45

McElwain, M. W., \& Burgasser, A. J. 2006, AJ, 132, 2074

McLean, I. S., Becklin, E. E., Bendiksen, O., Brims, G., Canfield, J., Figer, D. F., Graham, J. R., Hare, J., Lacayanga, F., Larkin, J. E., Larson, S. B., Levenson, N., Magnone, N. Teplitz, H., \& Wong, W. 1998, in Society of Photo-Optical Instrumentation Engineers (SPIE) Conference Series, Vol. 3354, Society of Photo-Optical Instrumentation Engineers (SPIE) Conference Series, ed. A. M. Fowler, 566-578

McLean, I. S., Prato, L., McGovern, M. R., Burgasser, A. J., Kirkpatrick, J. D., Rice, E. L., \& Kim, S. S. 2007, ApJ, 658, 1217

Meibom, S., \& Mathieu, R. D. 2005, ApJ, 620, 970

Moeckel, N., \& Bate, M. R. 2010, MNRAS, 404, 721

Mohanty, S., \& Basri, G. 2003, ApJ, 583, 451

Mohanty, S., Basri, G., Shu, F., Allard, F., \& Chabrier, G. 2002, ApJ, 571, 469

Phan-Bao, N., Forveille, T., Martín, E. L., \& Delfosse, X. 2006, ApJ, 645, L153

Prato, L., Simon, M., Mazeh, T., McLean, I. S., Norman, D., \& Zucker, S. 2002, ApJ, 569, 863

Radigan, J., Lafrenière, D., Jayawardhana, R., \& Doyon, R. 2009, ApJ, 698, 405

Raghavan, D., McAlister, H. A., Henry, T. J., Latham, D. W., Marcy, G. W., Mason, B. D., Gies, D. R., White, R. J., \& ten Brummelaar, T. A. 2010, ApJS, 190, 1

Ralchenko, Y., Kramida, A., Reader, J., \& NIST ASD Team. 2011, NIST Atomic Spectra Database (version 4.1)

Rayner, J. T., Toomey, D. W., Onaka, P. M., Denault, A. J., Stahlberger, W. E., Vacca, W. D., Cushing, M. C., \& Wang, S. 2003, PASP, 115, 362

Reid, I. N., Cruz, K. L., Kirkpatrick, J. D., Allen, P. R., Mungall, F., Liebert, J., Lowrance, P., \& Sweet, A. 2008, AJ, 136, 1290

Reid, I. N., \& Gizis, J. E. 1997, AJ, 113, 2246
Reid, I. N., Gizis, J. E., Kirkpatrick, J. D., \& Koerner, D. W. 2001, AJ, 121, 489

Reid, I. N., Hawley, S. L., \& Gizis, J. E. 1995, AJ, 110, 1838

Reid, I. N., Lewitus, E., Burgasser, A. J., \& Cruz, K. L. 2006 , ApJ, 639, 1114

Rice, E. L., Barman, T., Mclean, I. S., Prato, L., \& Kirkpatrick, J. D. 2010, ApJS, 186, 63

Rodler, F., Deshpande, R., Zapatero Osorio, M. R., Martín, E. L., Montgomery, M. M., Del Burgo, C., \& Creevey, O. L. 2012, A\&A, 538, A141

Rousselot, P., Lidman, C., Cuby, J.-G., Moreels, G., \& Monnet, G. 2000, A\&A, 354, 1134

Ruiz, M. T., Leggett, S. K., \& Allard, F. 1997, ApJ, 491, L107+ Salim, S., \& Gould, A. 2003, ApJ, 582, 1011

Saumon, D., \& Marley, M. S. 2008, ApJ, 689, 1327

Schmidt, S. J., West, A. A., Hawley, S. L., \& Pineda, J. S. 2010 AJ, 139, 1808

Seifahrt, A., Guenther, E., \& Neuhäuser, R. 2005, A\&A, 440, 967 Seifahrt, A., Röll, T., Neuhäuser, R., Reiners, A., Kerber, F., Käufl, H. U., Siebenmorgen, R., \& Smette, A. 2008, A\&A, 484, 429

Shatsky, N., \& Tokovinin, A. 2002, A\&A, 382, 92

Sheppard, S. S., \& Cushing, M. C. 2009, AJ, 137, 304

Siegler, N., Close, L. M., Burgasser, A. J., Cruz, K. L., Marois, C., Macintosh, B., \& Barman, T. 2007, AJ, 133, 2320

Silvestri, N. M., Hawley, S. L., West, A. A., Szkody, P., Bochanski, J. J., Eisenstein, D. J., McGehee, P., Schmidt, G. D., Smith, J. A., Wolfe, M. A., Harris, H. C., Kleinman, S. J., Liebert, J., Nitta, A., Barentine, J. C., Brewington, H. J., Brinkmann, J., Harvanek, M., Krzesiński, J., Long, D., Neilsen, Jr., E. H., Schneider, D. P., \& Snedden, S. A. 2006, AJ, 131, 1674

Simons, D. A., \& Tokunaga, A. 2002, PASP, 114, 169

Skrutskie, M. F., et al. 2006, AJ, 131, 1163

Socrates, A., Katz, B., Dong, S., \& Tremaine, S. 2012, ApJ, 750, 106

Stamatellos, D., Maury, A., Whitworth, A., \& André, P. 2011 MNRAS, 413, 1787

Stamatellos, D., \& Whitworth, A. P. 2008, A\&A, 480, 879

-. 2009, MNRAS, 392, 413

Stassun, K. G., Mathieu, R. D., \& Valenti, J. A. 2006, Nature, 440,311

Stephens, D. C., Leggett, S. K., Cushing, M. C., Marley, M. S., Saumon, D., Geballe, T. R., Golimowski, D. A., Fan, X., \& Noll, K. S. 2009, ApJ, 702, 154

Sterzik, M. F., \& Durisen, R. H. 2003, A\&A, 400, 1031

Sterzik, M. F., \& Tokovinin, A. A. 2002, A\&A, 384, 1030

Stumpf, M. B., Brandner, W., Henning, T., Bouy, H., Koehler, R., Hormuth, F., Joergens, V., \& Kasper, M. 2008, Astronomy and Astrophysics, submitted

Tody, D. 1986, in Society of Photo-Optical Instrumentation Engineers (SPIE) Conference Series, Vol. 627, Society of Photo-Optical Instrumentation Engineers (SPIE) Conference Series, ed. D. L. Crawford, 733-+

Tokovinin, A. 2008, MNRAS, 389, 925

Tokovinin, A., Thomas, S., Sterzik, M., \& Udry, S. 2006, A\&A, 450,681

Tokovinin, A. A. 1997, A\&AS, 124, 75

Tokunaga, A. T., Simons, D. A., \& Vacca, W. D. 2002, PASP, 114,180

Vacca, W. D., Cushing, M. C., \& Rayner, J. T. 2003, PASP, 115, 389

Walkowicz, L. M., Hawley, S. L., \& West, A. A. 2004, PASP, 116, 1105

Weinberg, M. D., Shapiro, S. L., \& Wasserman, I. 1987, ApJ, 312, 367

West, A. A., \& Basri, G. 2009, ApJ, 693, 1283

West, A. A., Bochanski, J. J., Hawley, S. L., Cruz, K. L., Covey, K. R., Silvestri, N. M., Reid, I. N., \& Liebert, J. 2006, AJ, 132 , 2507

West, A. A., \& Hawley, S. L. 2008, PASP, 120, 1161

West, A. A., Hawley, S. L., Bochanski, J. J., Covey, K. R., Reid, I. N., Dhital, S., Hilton, E. J., \& Masuda, M. 2008, AJ, 135, 785 West, A. A., Hawley, S. L., Walkowicz, L. M., Covey, K. R., Silvestri, N. M., Raymond, S. N., Harris, H. C., Munn, J. A., McGehee, P. M., Ivezić, Ž., \& Brinkmann, J. 2004, AJ, 128, 426 
West, A. A., Walkowicz, L. M., \& Hawley, S. L. 2005, PASP, 117, 706
Wilson, J. C., Miller, N. A., Gizis, J. E., Skrutskie, M. F., Houck, J. R., Kirkpatrick, J. D., Burgasser, A. J., \& Monet, D. G.

2003, in IAU Symposium, Vol. 211, Brown Dwarfs, ed.

E. Martín, 197-+

York, D. G., et al. 2000, AJ, 120, 1579

Zapatero Osorio, M. R., Martín, E. L., Béjar, V. J. S., Bouy, H., Deshpande, R., \& Wainscoat, R. J. 2007, ApJ, 666, 1205 